\title{
Hemicryptophanes with Improved Fluorescent Properties for the Selective Recognition of Acetylcholine over Choline
}

Augustin Long,${ }^{\dagger}$ Elise Antonetti,${ }^{\dagger}$ Alberto Insuasty,${ }^{\dagger}$ Sandra Pinet, ${ }^{\S}$ Isabelle Gosse,${ }^{\S}$ Vincent Robert, ${ }^{\perp}$ Jean-Pierre Dutasta, ${ }^{\dagger}$ and Alexandre Martinez ${ }^{* \dagger}$

† Aix-Marseille Univ., CNRS, Centrale Marseille, iSm2, UMR 7113, 13397, Marseille, France

$\S$ ISM, UMR 5255 CNRS, Bordeaux INP and Univ. Bordeaux, 351 cours de la Libération, F-33400 Talence, France.

${ }^{\perp}$ Laboratoire de Chimie Quantique Institut de Chimie, UMR CNRS 7177, Université de Strasbourg, 4, rue Blaise Pascal, F-67070 Strasbourg, France.

\$ Laboratoire de Chimie, Ecole Normale Supérieure de Lyon, CNRS, UCBL, 46 allée d'Italie, F-69364 Lyon, France 


\section{Table of contents}

1. NMR and mass spectra ..................................................................................3

2. Photophysical studies ....................................................................................18

2.1. Spectra and properties .................................................................... 18

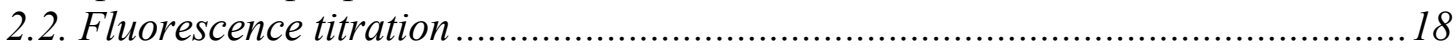

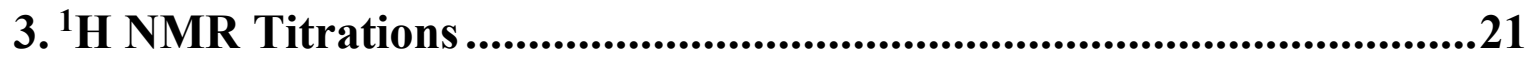

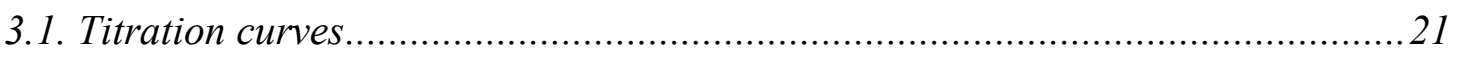

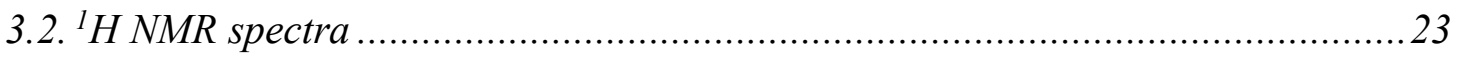

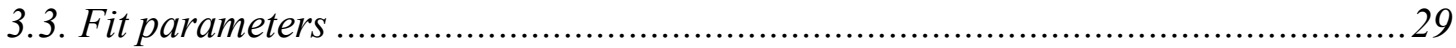

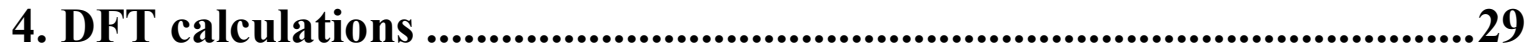

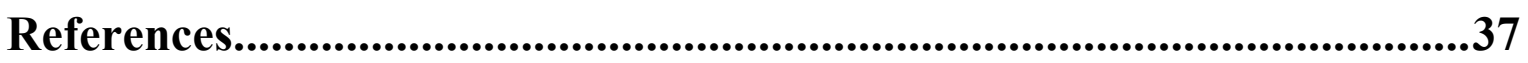




\section{NMR and mass spectra}

।

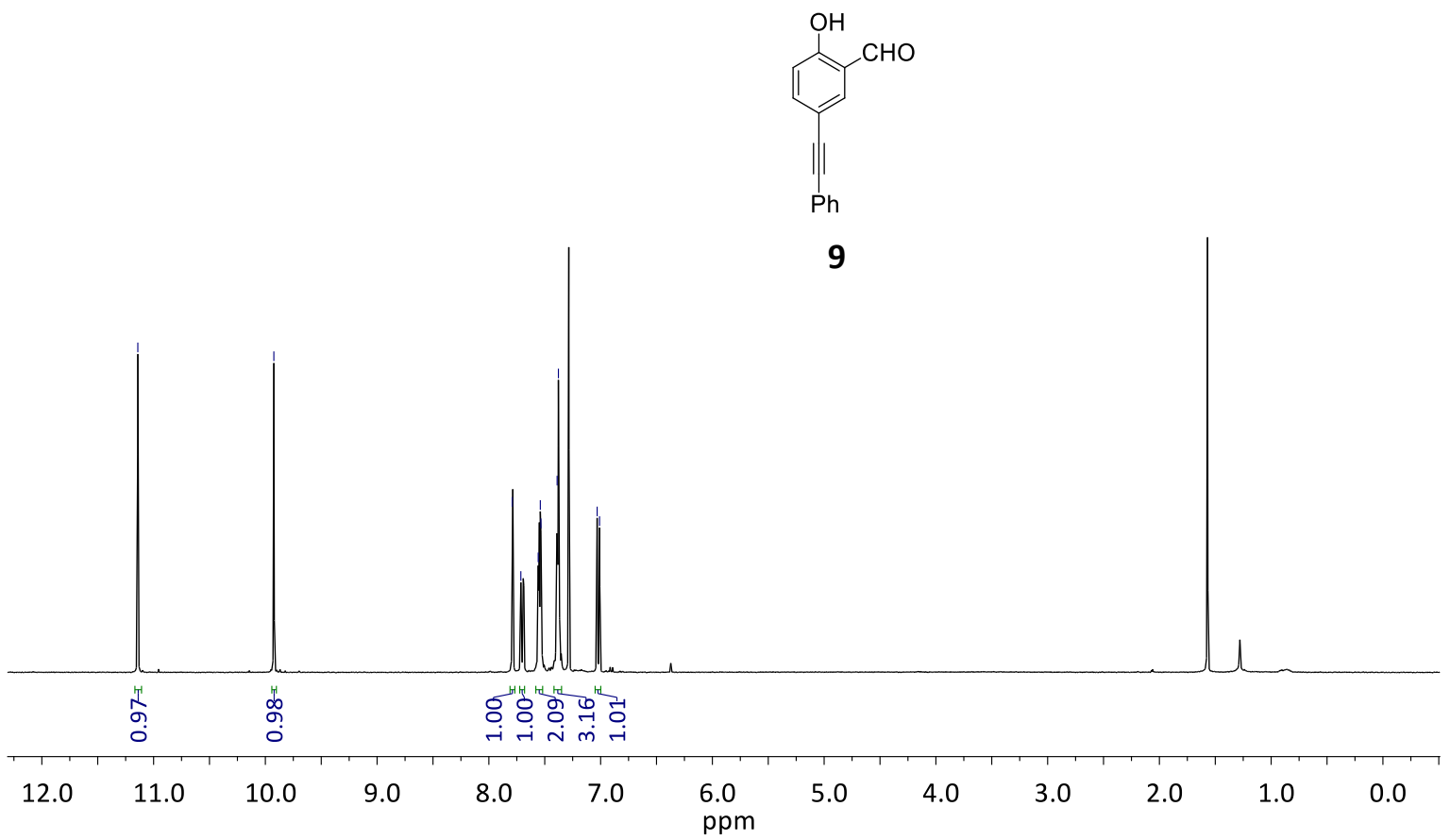

Figure S1. ${ }^{1} \mathrm{H}$ NMR Spectrum of $9\left(\mathrm{CDCl}_{3}, 400 \mathrm{MHz}, 298 \mathrm{~K}\right)$.

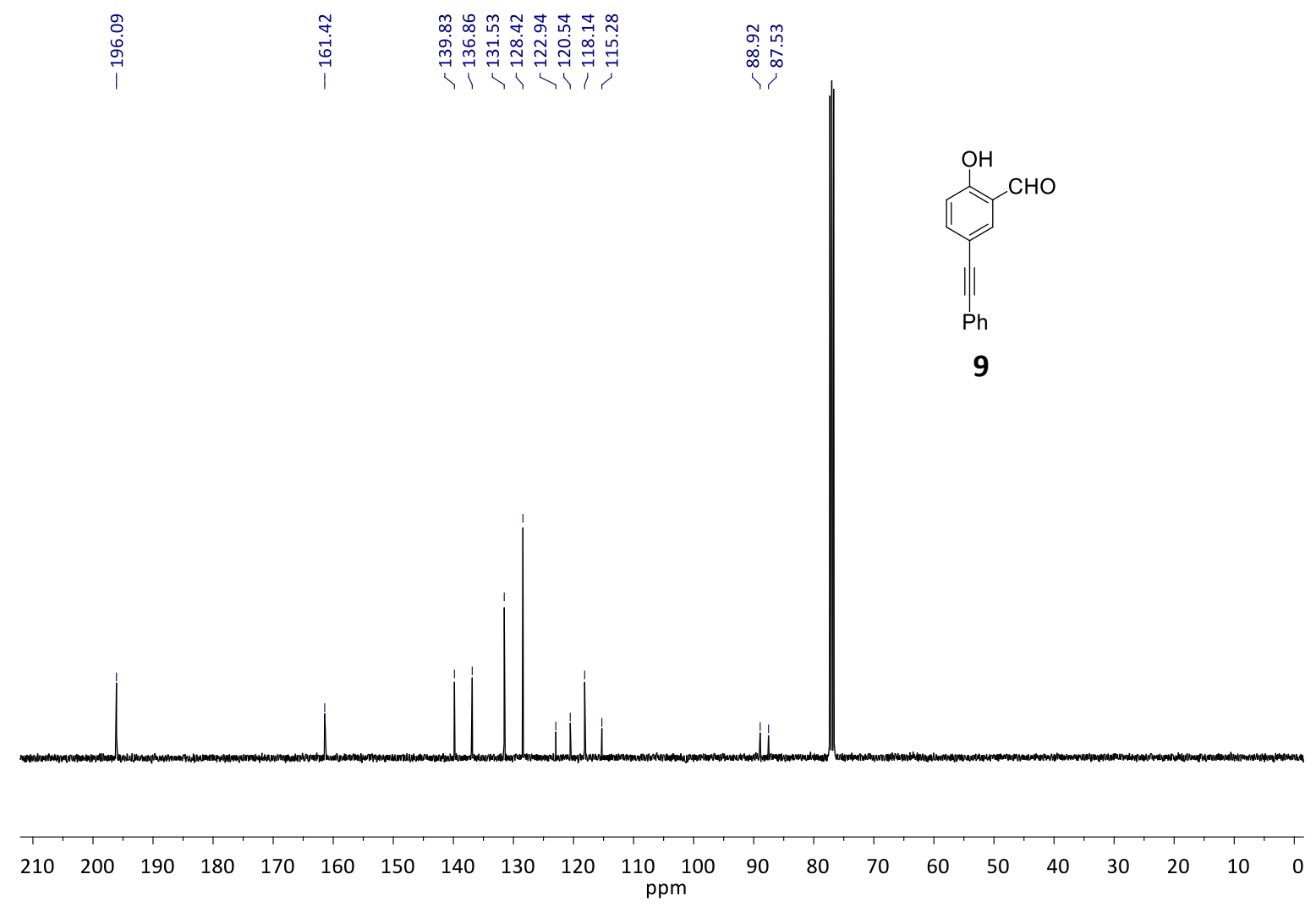

Figure S2. ${ }^{13} \mathrm{C}\left\{{ }^{1} \mathrm{H}\right\}$ NMR Spectrum of $9\left(\mathrm{CDCl}_{3}, 100 \mathrm{MHz}, 298 \mathrm{~K}\right)$. 


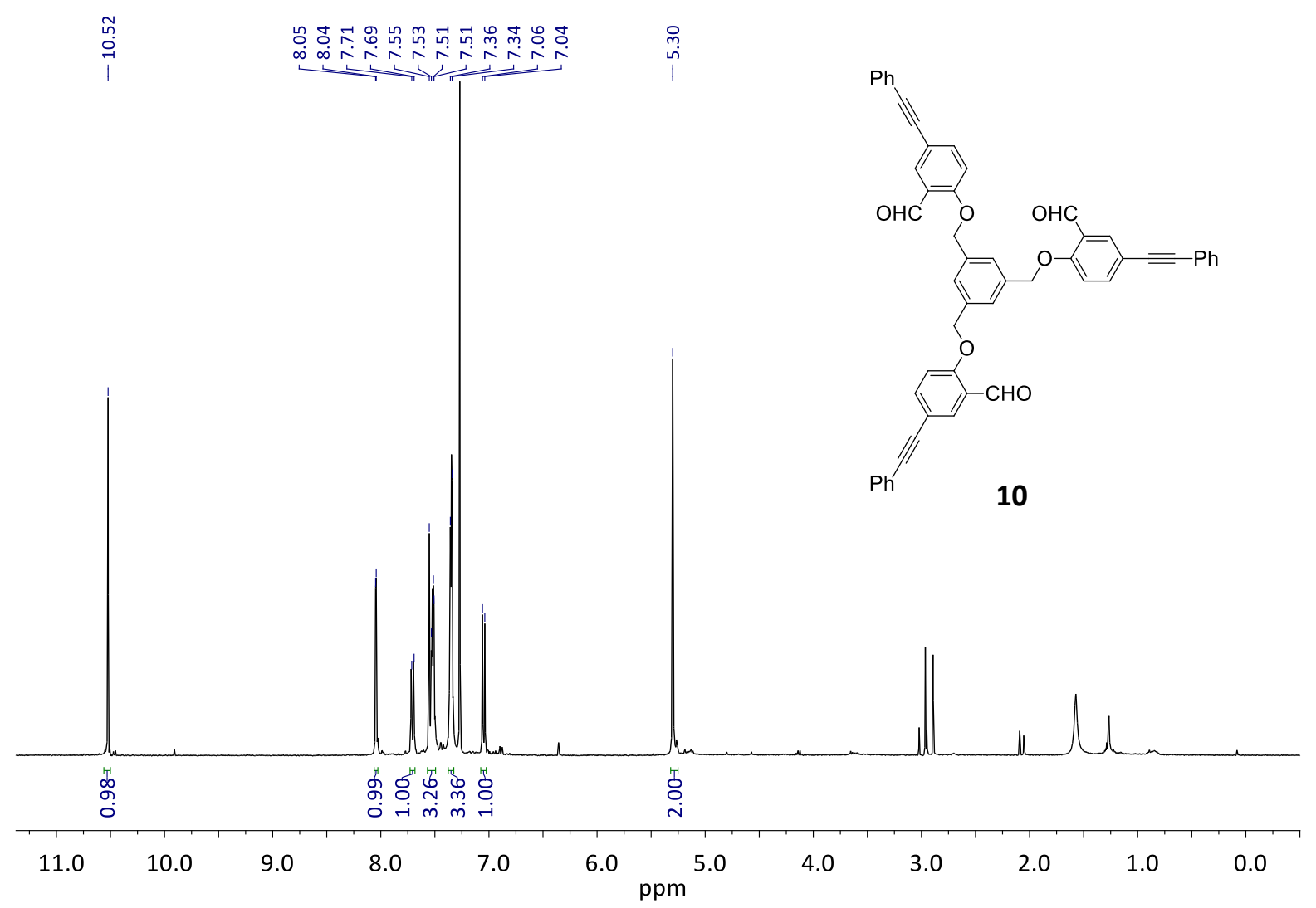

Figure S3. ${ }^{1} \mathrm{H}$ NMR Spectrum of $10\left(\mathrm{CDCl}_{3}, 400 \mathrm{MHz}, 298 \mathrm{~K}\right)$.

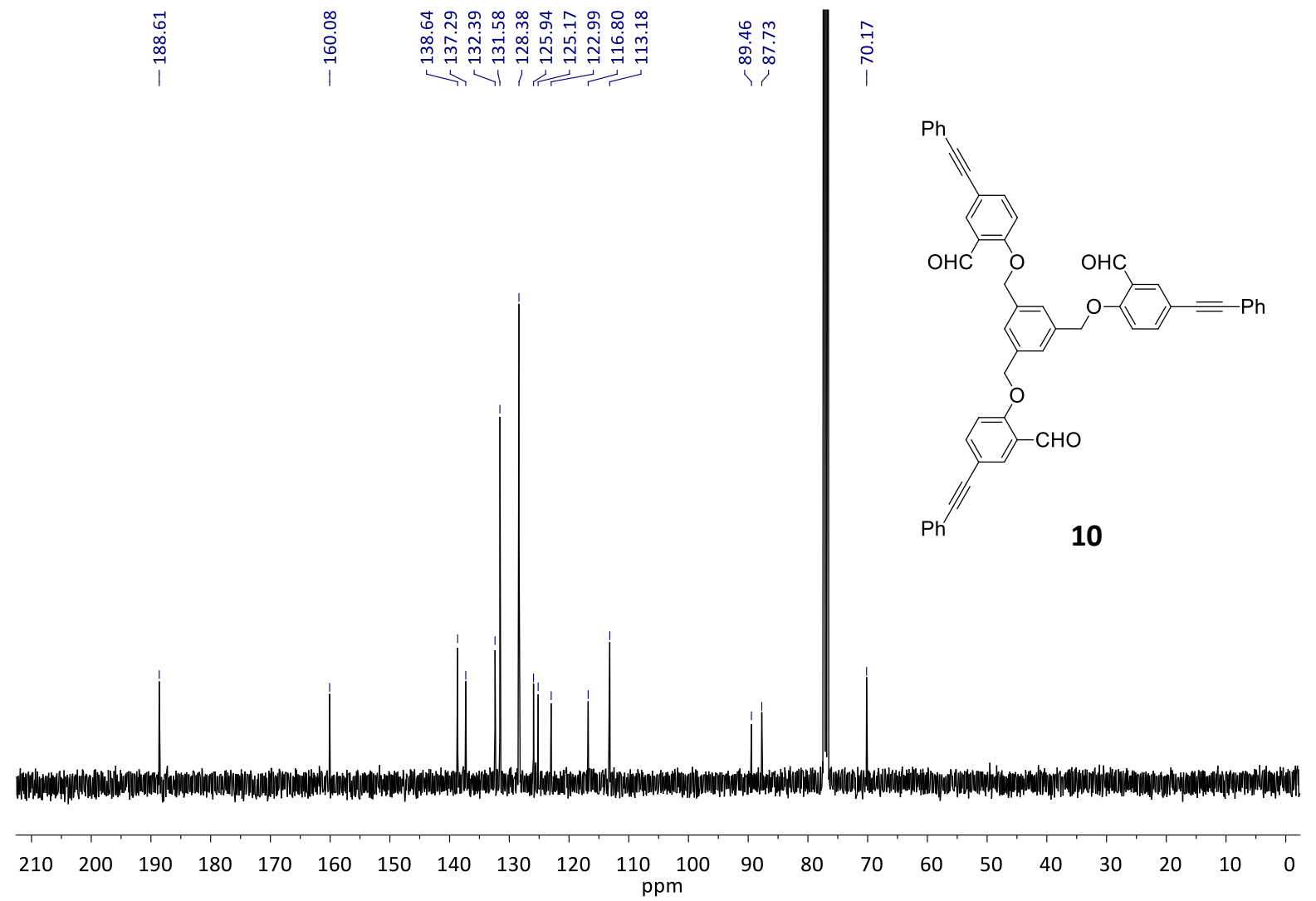

Figure S4. ${ }^{13} \mathrm{C}\left\{{ }^{1} \mathrm{H}\right\}$ NMR Spectrum of $\mathbf{1 0}\left(\mathrm{CDCl}_{3}, 100 \mathrm{MHz}, 298 \mathrm{~K}\right)$. 


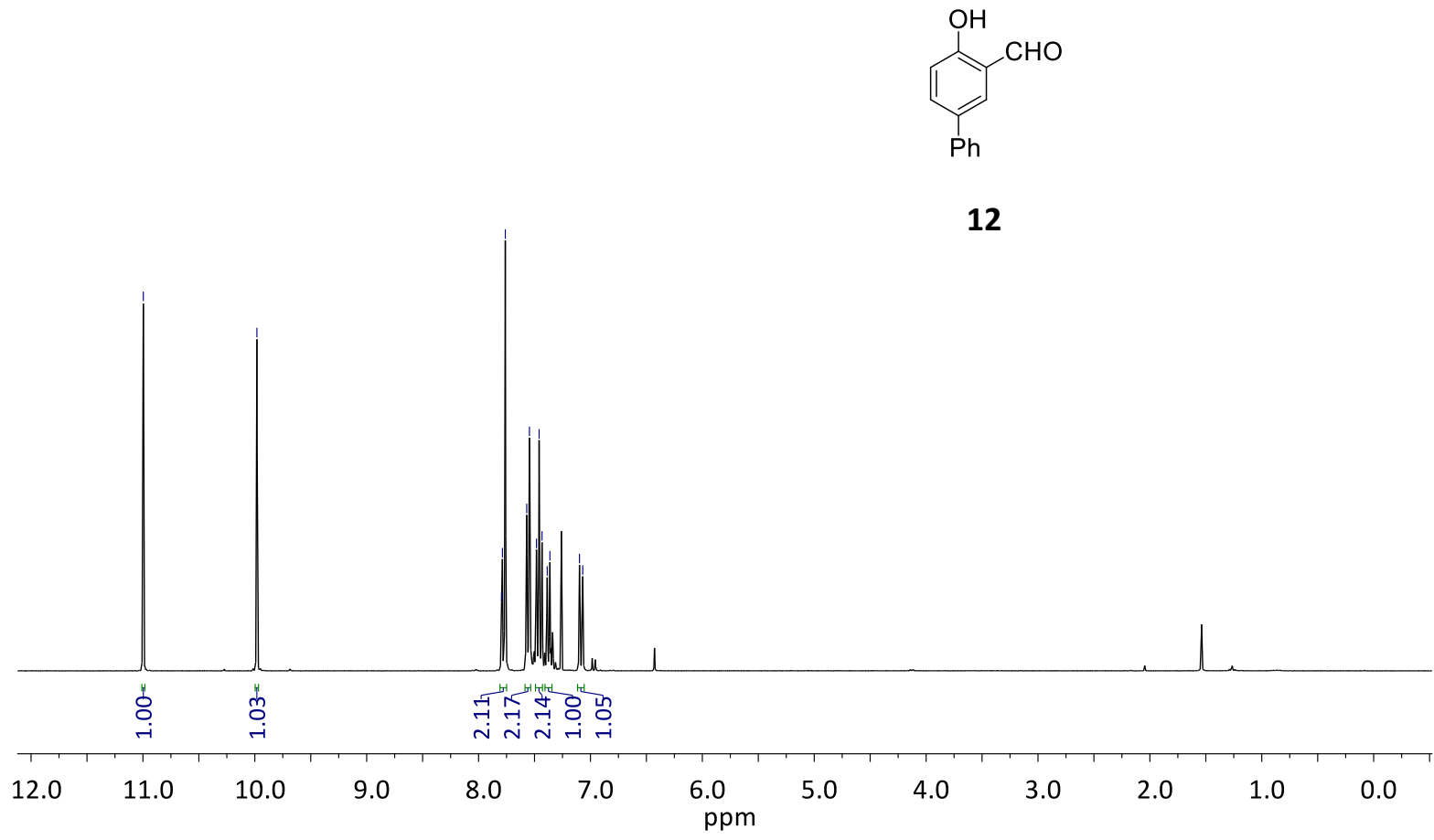

Figure S5. ${ }^{1} \mathrm{H}$ NMR Spectrum of $\mathbf{1 2}\left(\mathrm{CDCl}_{3}, 300 \mathrm{MHz}, 298 \mathrm{~K}\right)$.

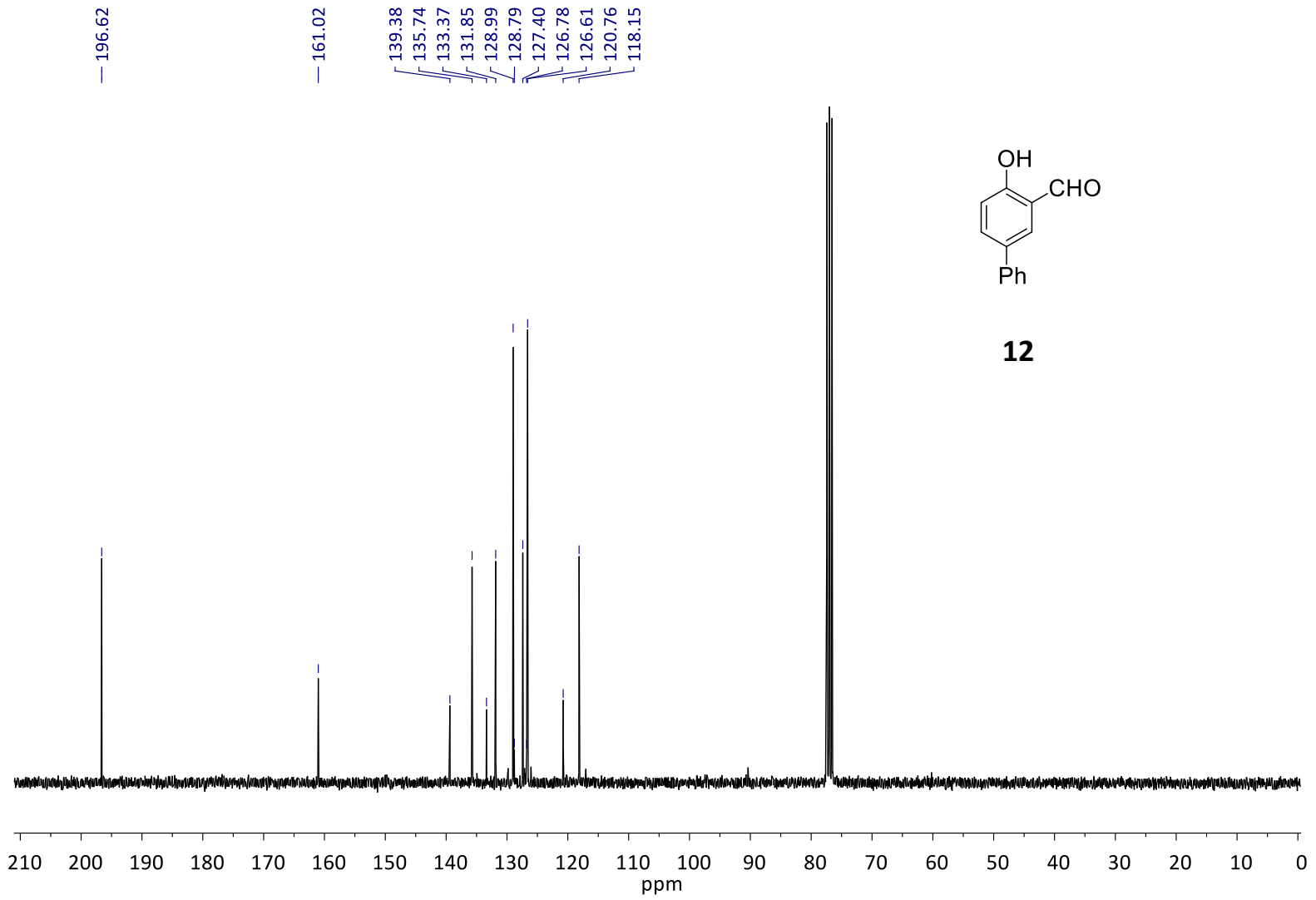

Figure S6. ${ }^{13} \mathrm{C}\left\{{ }^{1} \mathrm{H}\right\}$ NMR Spectrum of $12\left(\mathrm{CDCl}_{3}, 75 \mathrm{MHz}, 298 \mathrm{~K}\right)$. 


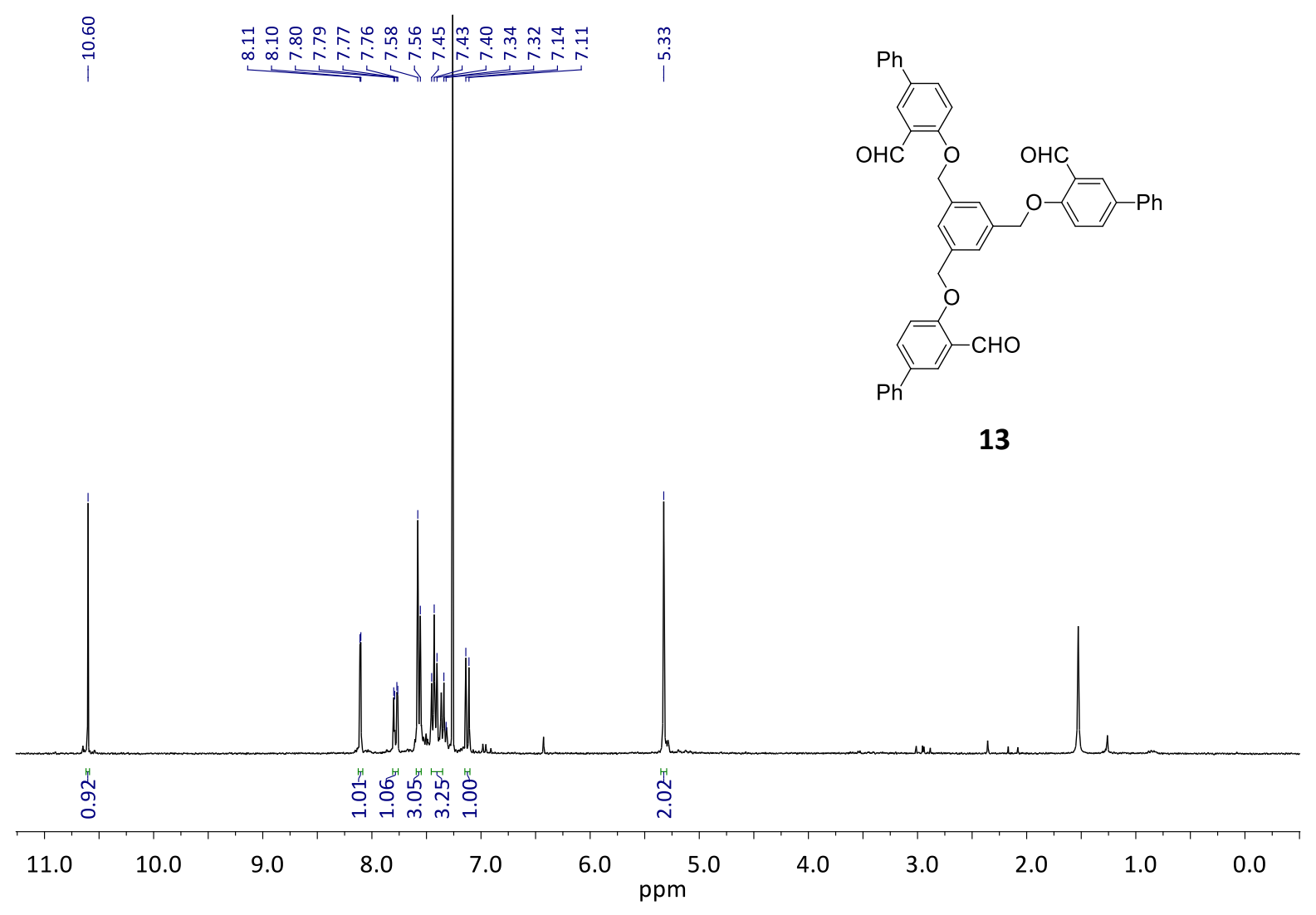

Figure S7. ${ }^{1} \mathrm{H}$ NMR Spectrum of $13\left(\mathrm{CDCl}_{3}, 100 \mathrm{MHz}, 298 \mathrm{~K}\right)$.

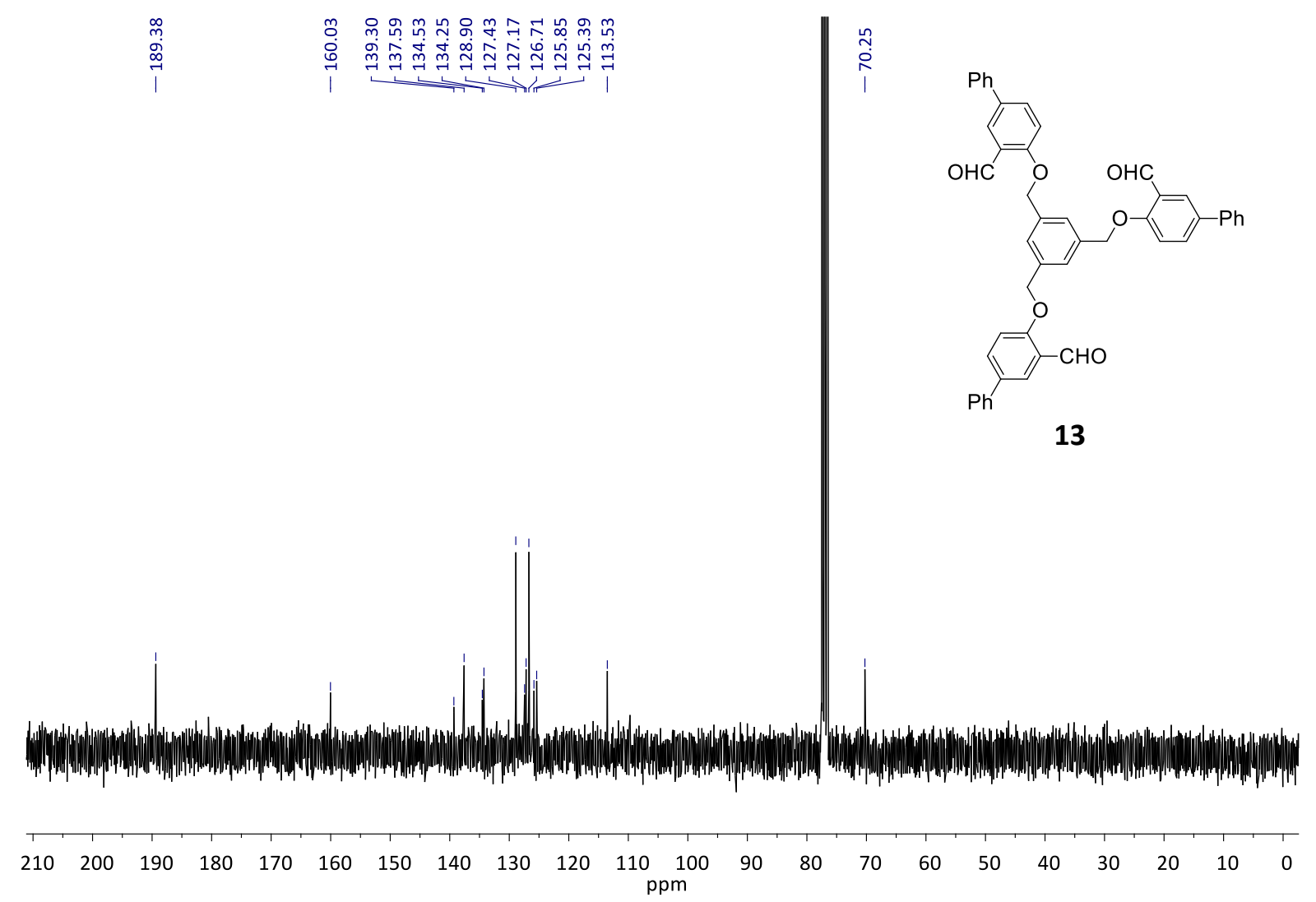

Figure S8. ${ }^{13} \mathrm{C}\left\{{ }^{1} \mathrm{H}\right\}$ NMR Spectrum of $13\left(\mathrm{CDCl}_{3}, 75 \mathrm{MHz}, 298 \mathrm{~K}\right)$. 


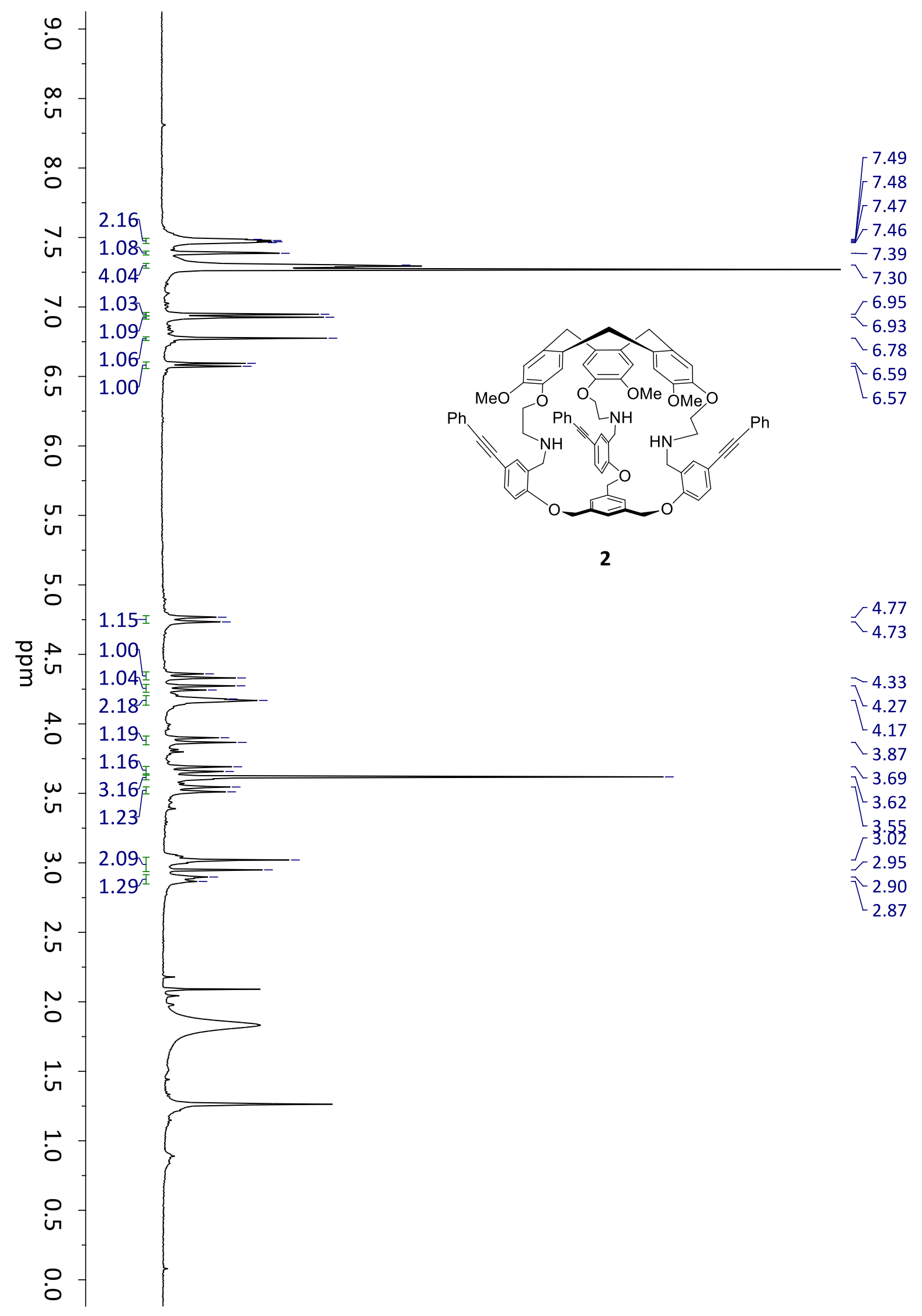

Figure S9. ${ }^{1} \mathrm{H}$ NMR Spectrum of $2\left(\mathrm{CDCl}_{3}, 400 \mathrm{MHz}, 298 \mathrm{~K}\right)$. 


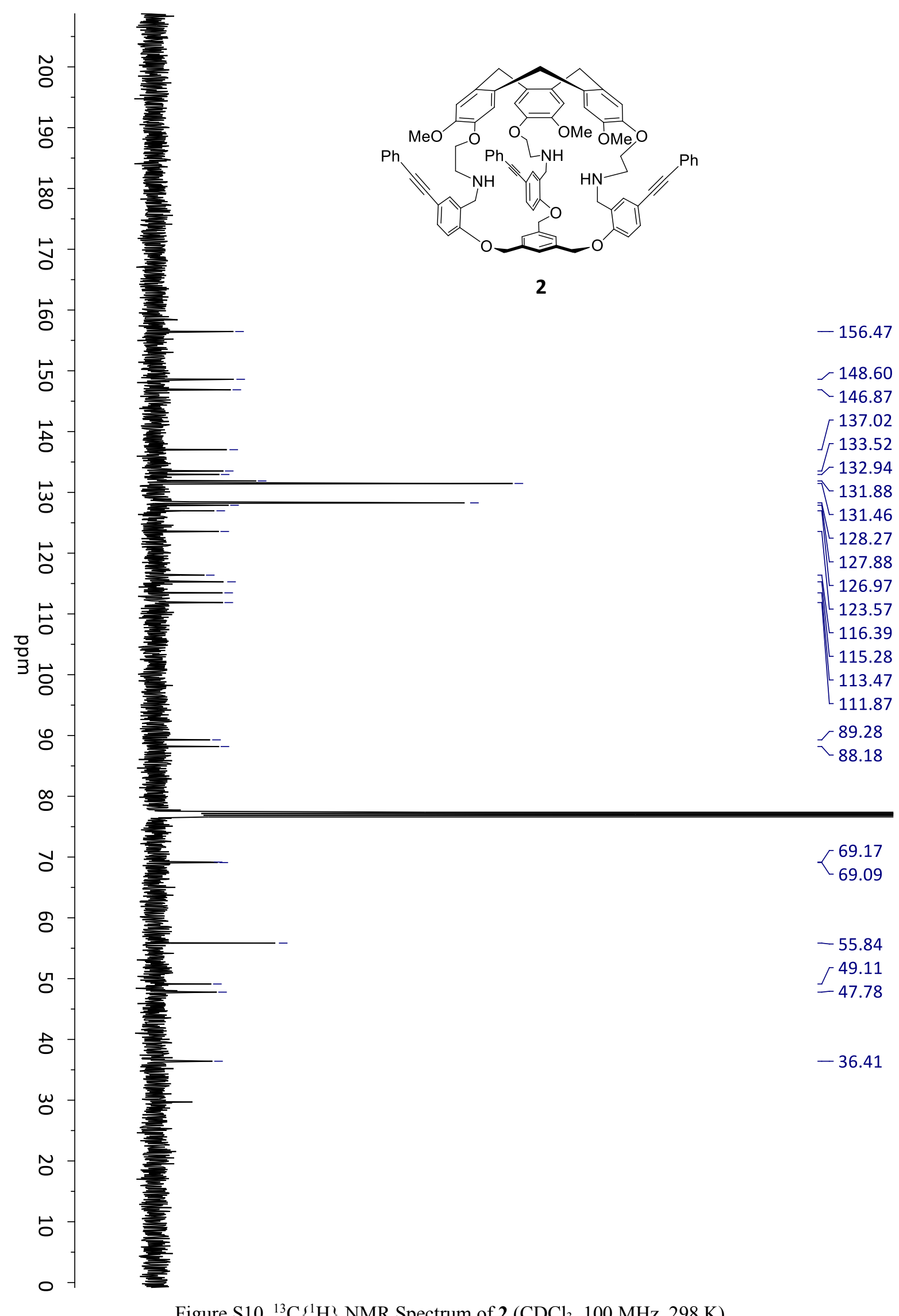

Figure S10. ${ }^{13} \mathrm{C}\left\{{ }^{1} \mathrm{H}\right\}$ NMR Spectrum of $2\left(\mathrm{CDCl}_{3}, 100 \mathrm{MHz}, 298 \mathrm{~K}\right)$. 


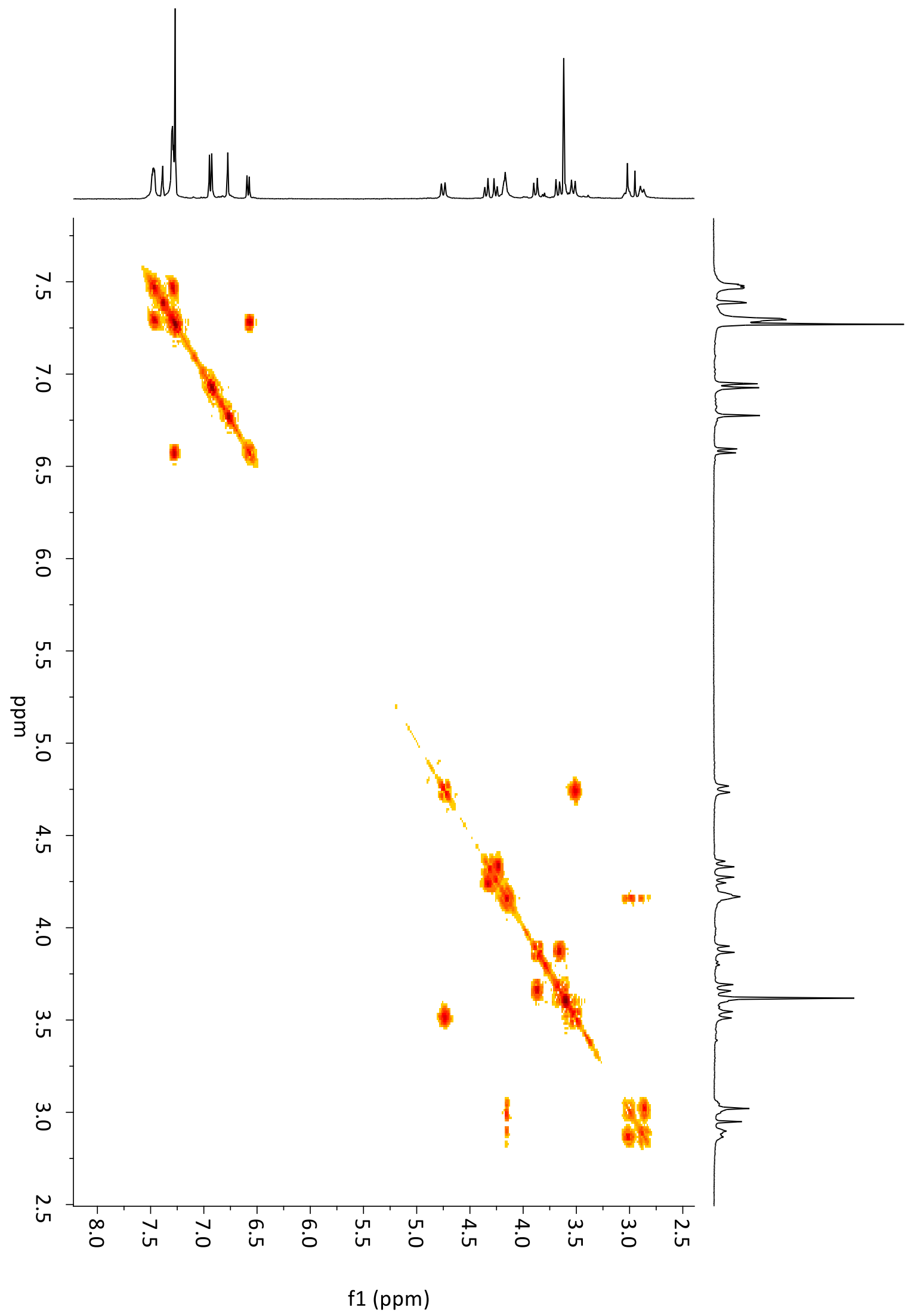

Figure S11. COSY Spectrum of $2\left(\mathrm{CDCl}_{3}, 400 \mathrm{MHz}, 298 \mathrm{~K}\right)$. 


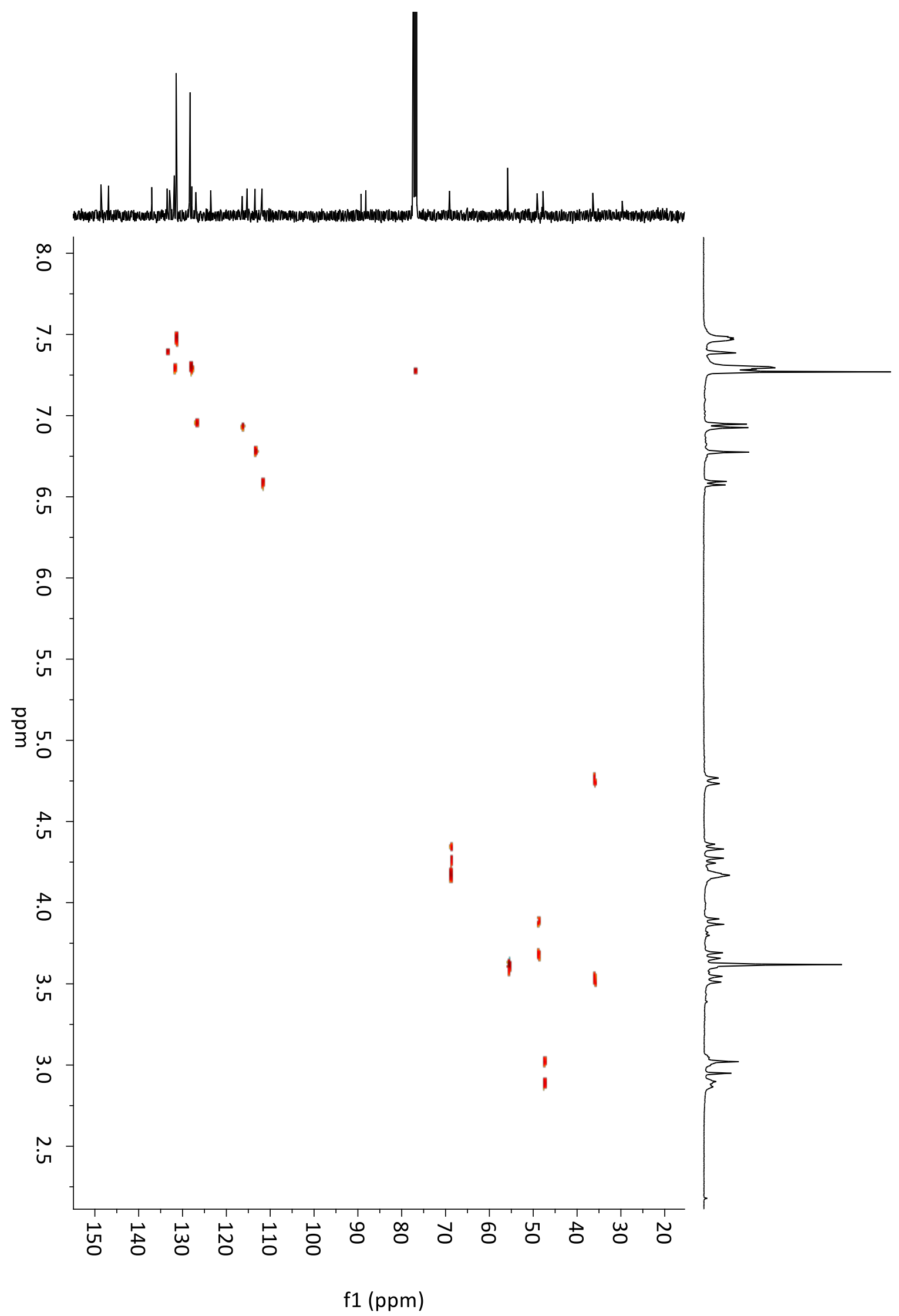

Figure S12. HSQC Spectrum of $2\left(\mathrm{CDCl}_{3}, 400 \mathrm{MHz}, 298 \mathrm{~K}\right)$. 


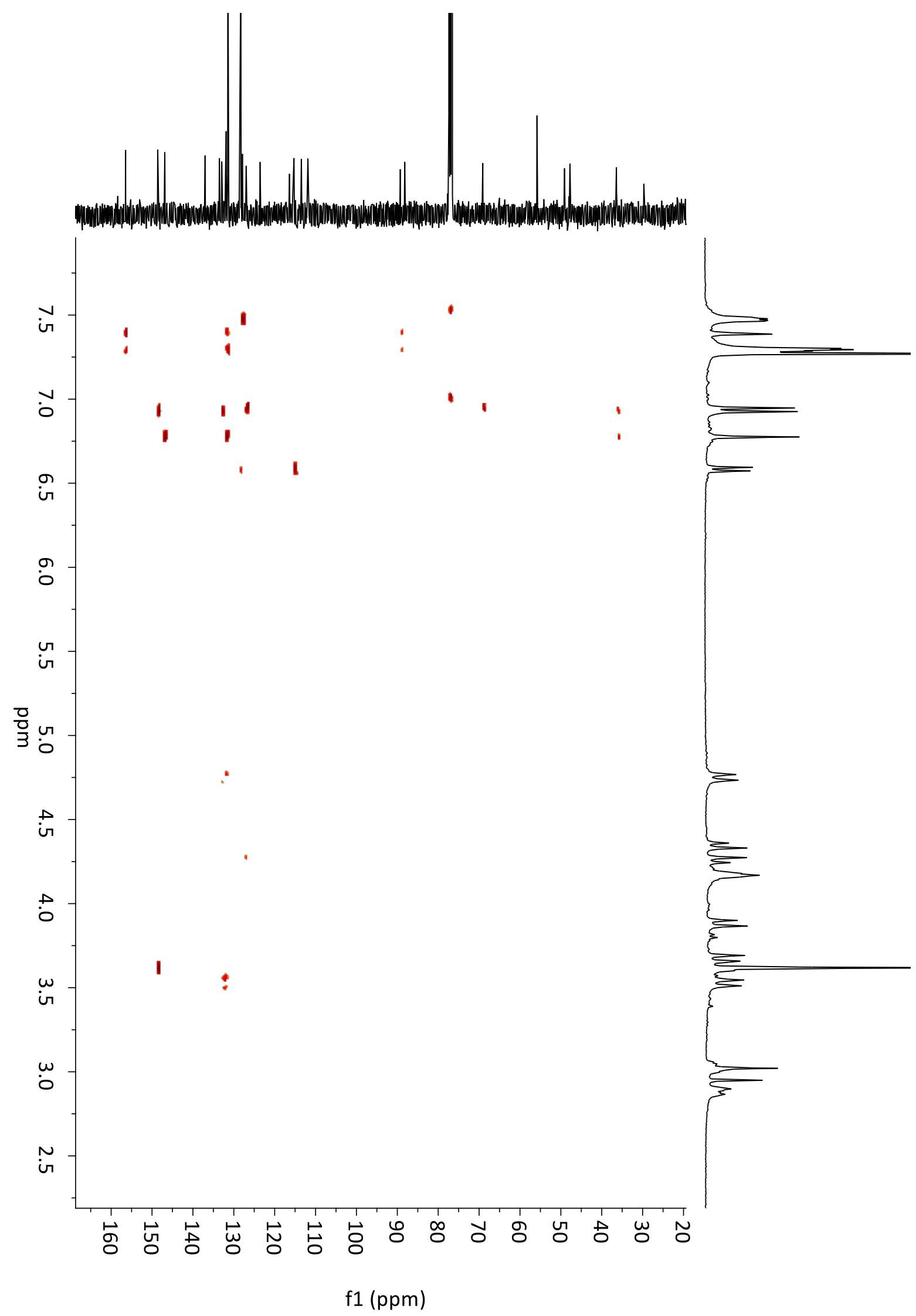

Figure S13. HMBC Spectrum of $2\left(\mathrm{CDCl}_{3}, 400 \mathrm{MHz}, 298 \mathrm{~K}\right)$. 


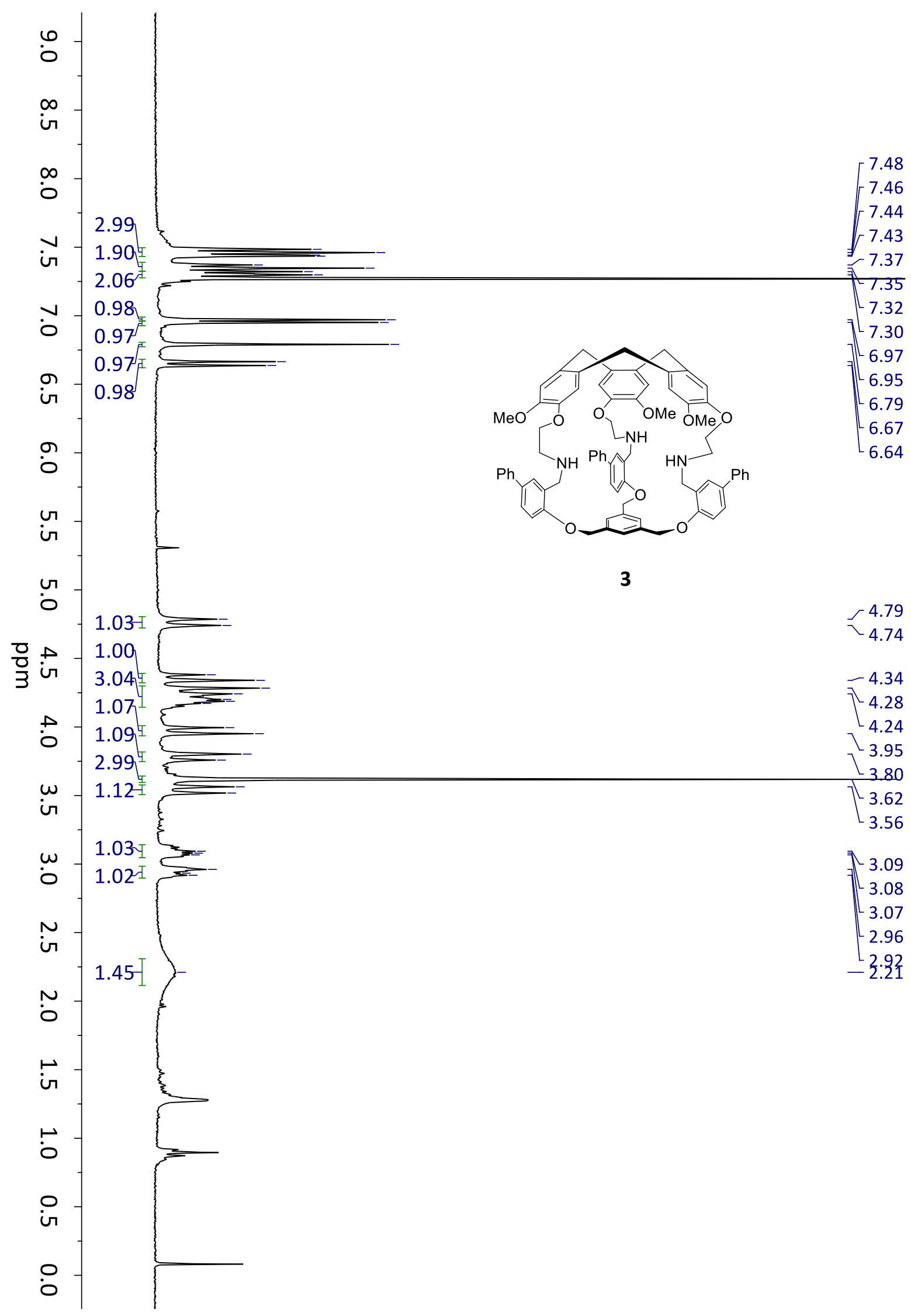

Figure S14. ${ }^{1} \mathrm{H}$ NMR Spectrum of $3\left(\mathrm{CDCl}_{3}, 400 \mathrm{MHz}, 298 \mathrm{~K}\right)$. 


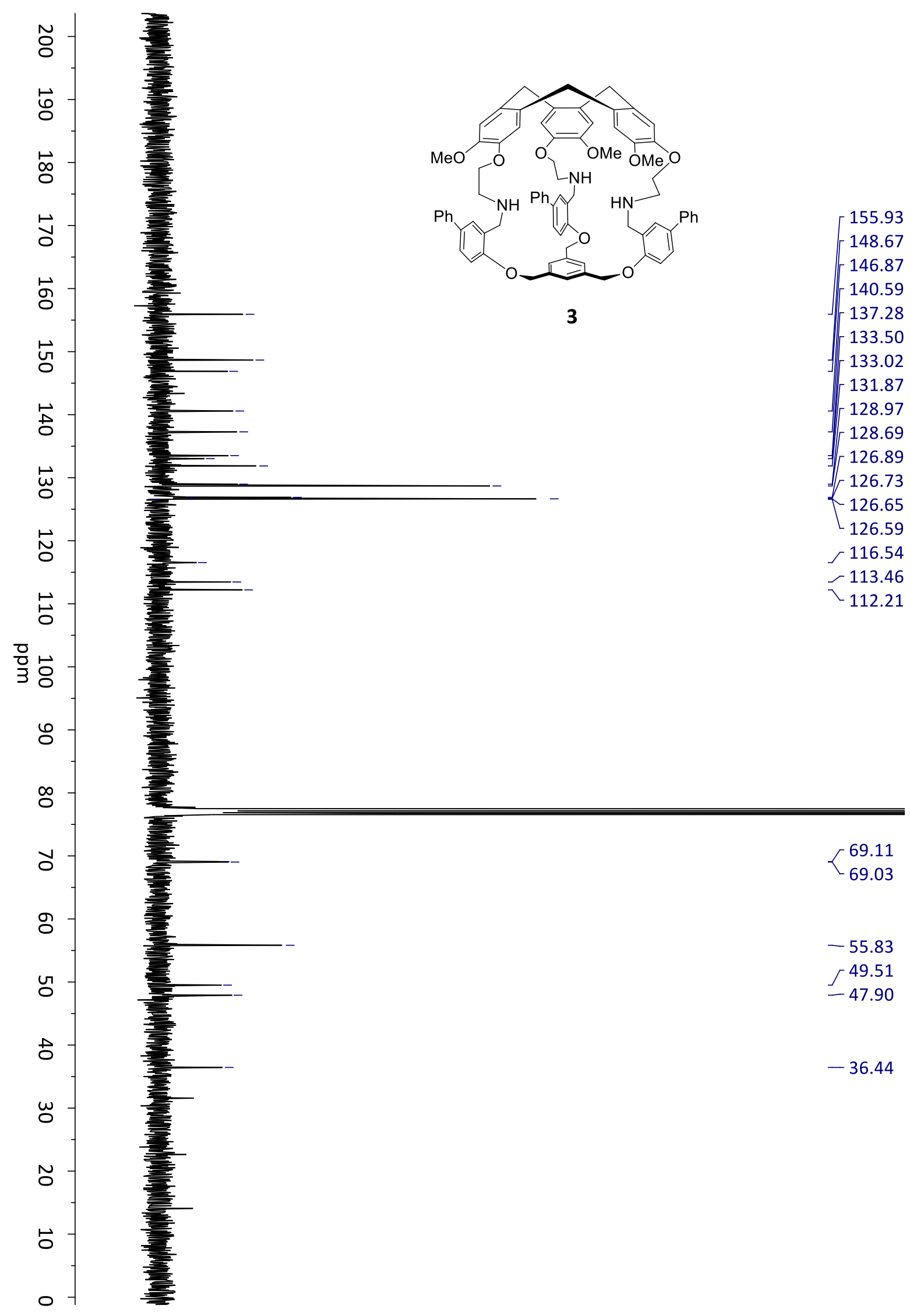

Figure S15. ${ }^{13} \mathrm{C}\left\{{ }^{1} \mathrm{H}\right\}$ NMR Spectrum of $\mathbf{3}\left(\mathrm{CDCl}_{3}, 100 \mathrm{MHz}, 298 \mathrm{~K}\right)$. 


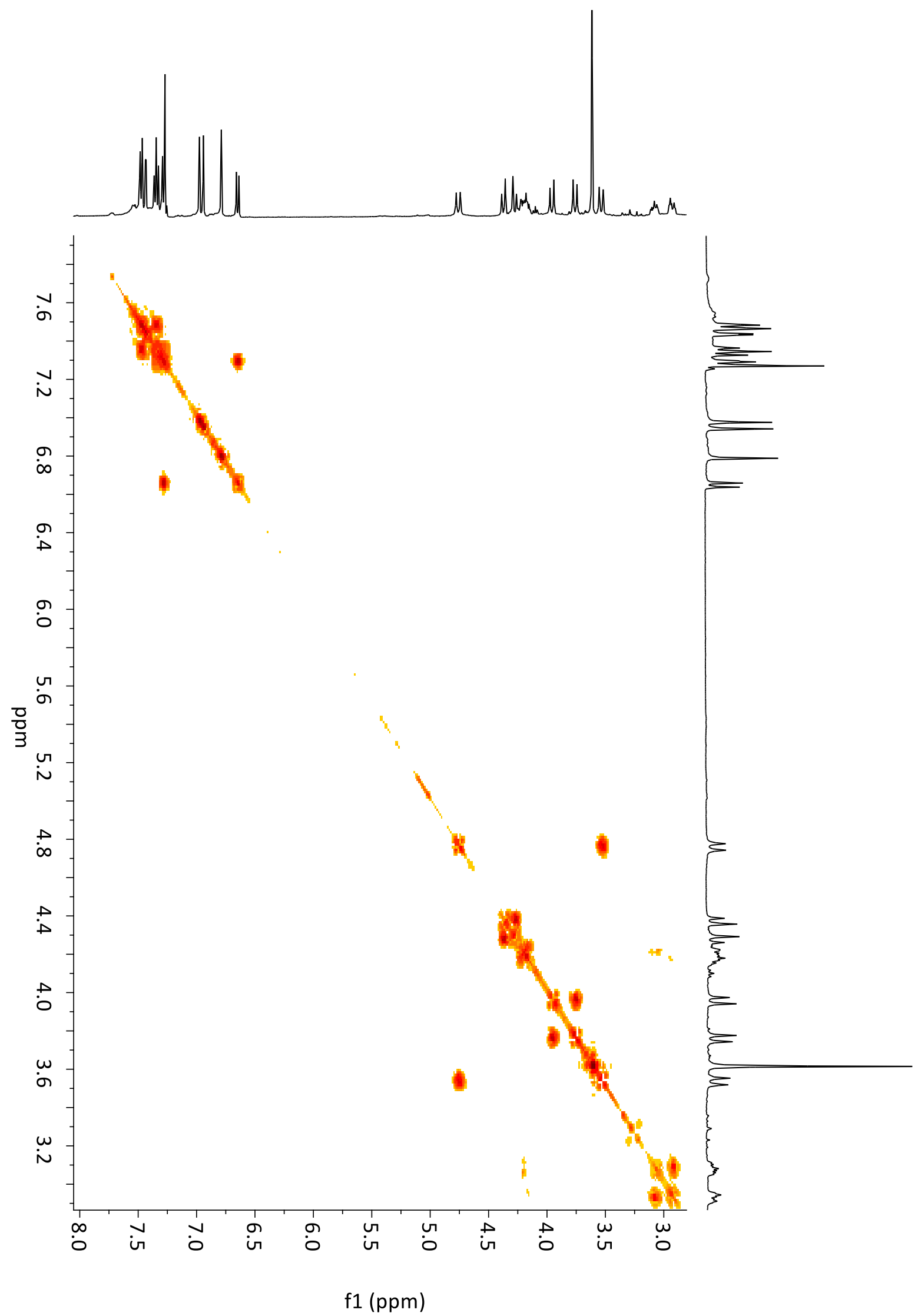

Figure S16. COSY Spectrum of $\mathbf{3}\left(\mathrm{CDCl}_{3}, 400 \mathrm{MHz}, 298 \mathrm{~K}\right)$. 


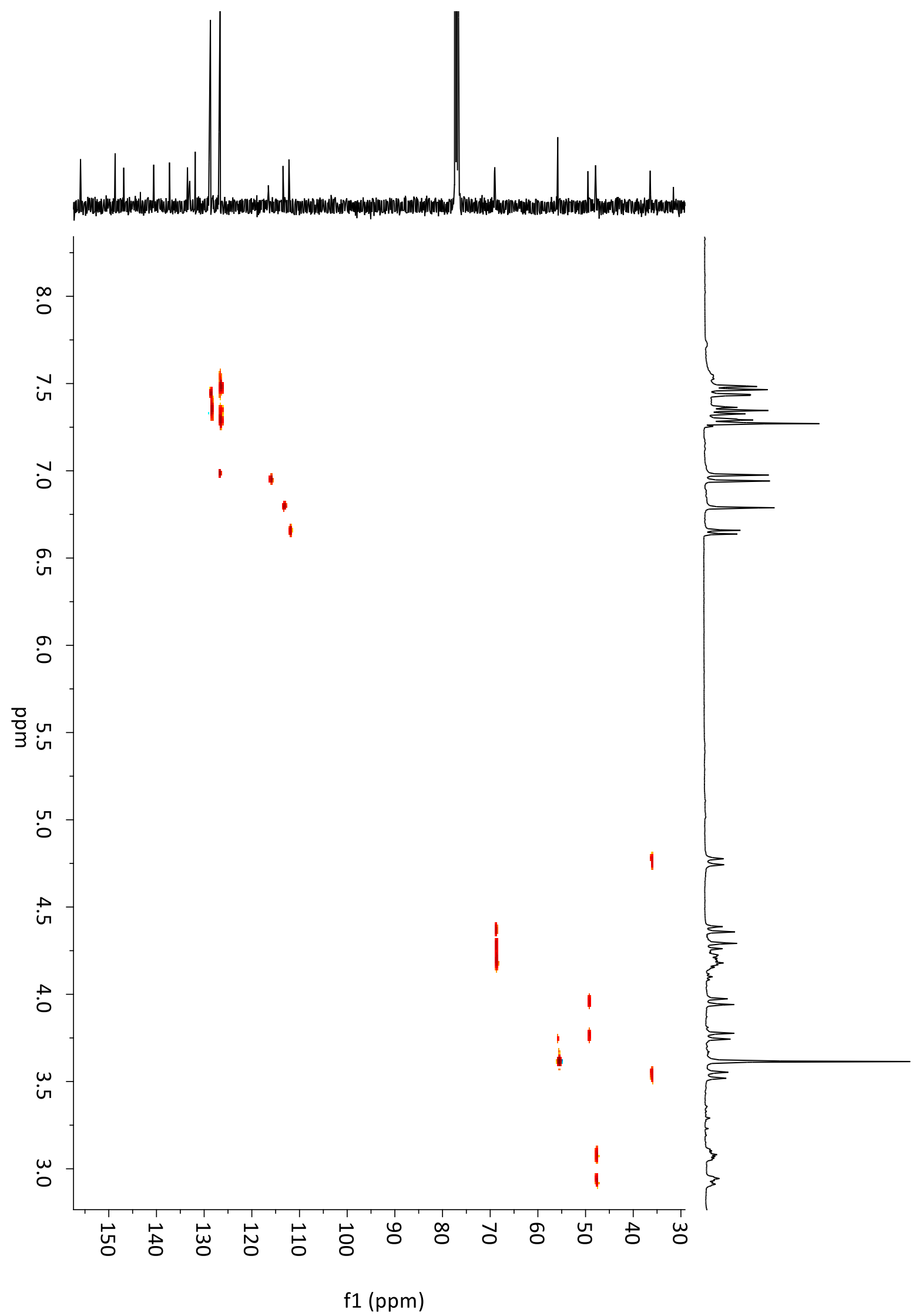

Figure S17. HSQC Spectrum of $3\left(\mathrm{CDCl}_{3}, 400 \mathrm{MHz}, 298 \mathrm{~K}\right)$. 


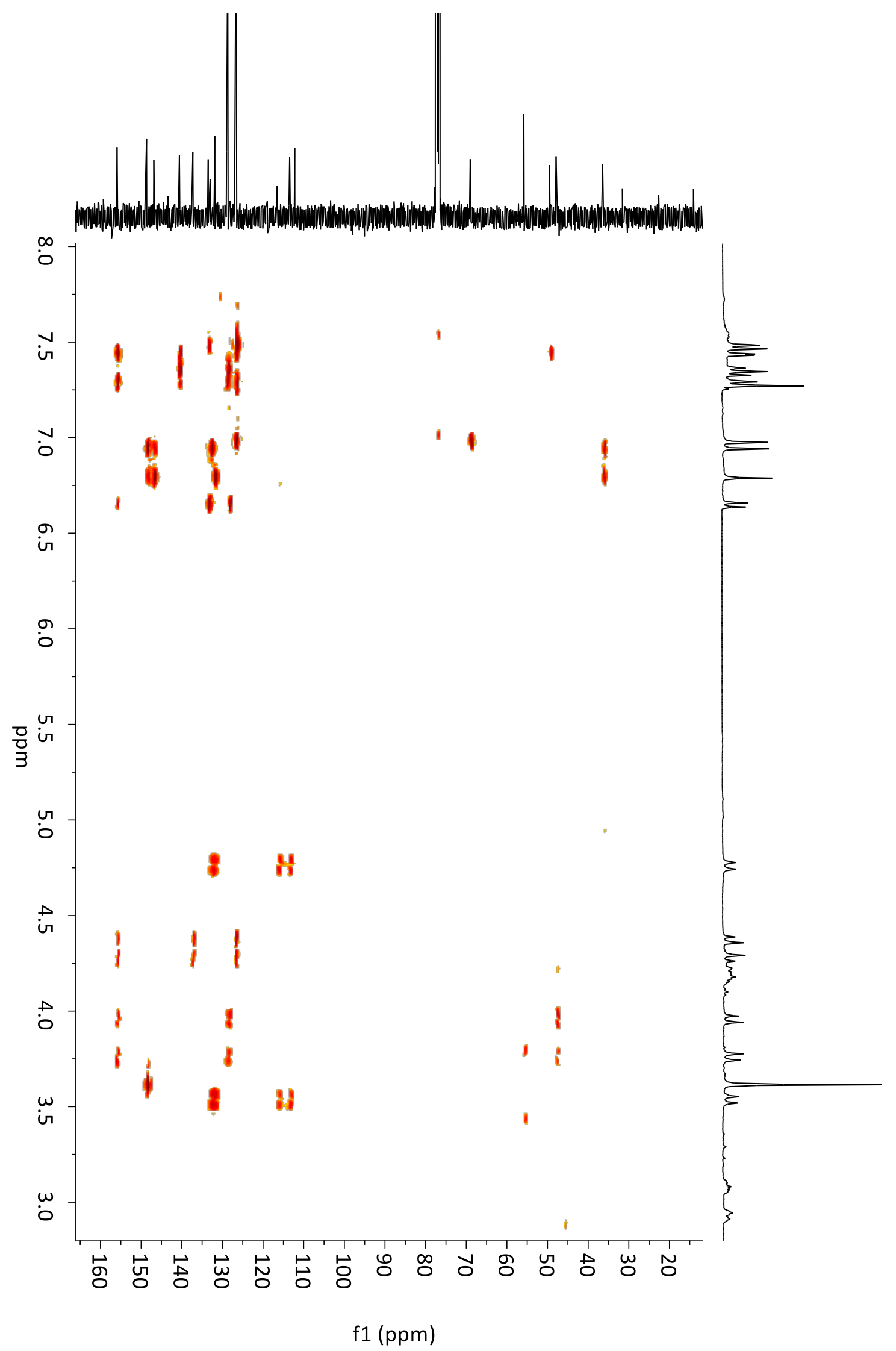

Figure S18. HMBC Spectrum of $\mathbf{3}\left(\mathrm{CDCl}_{3}, 400 \mathrm{MHz}, 298 \mathrm{~K}\right)$. 


\subsection{9}

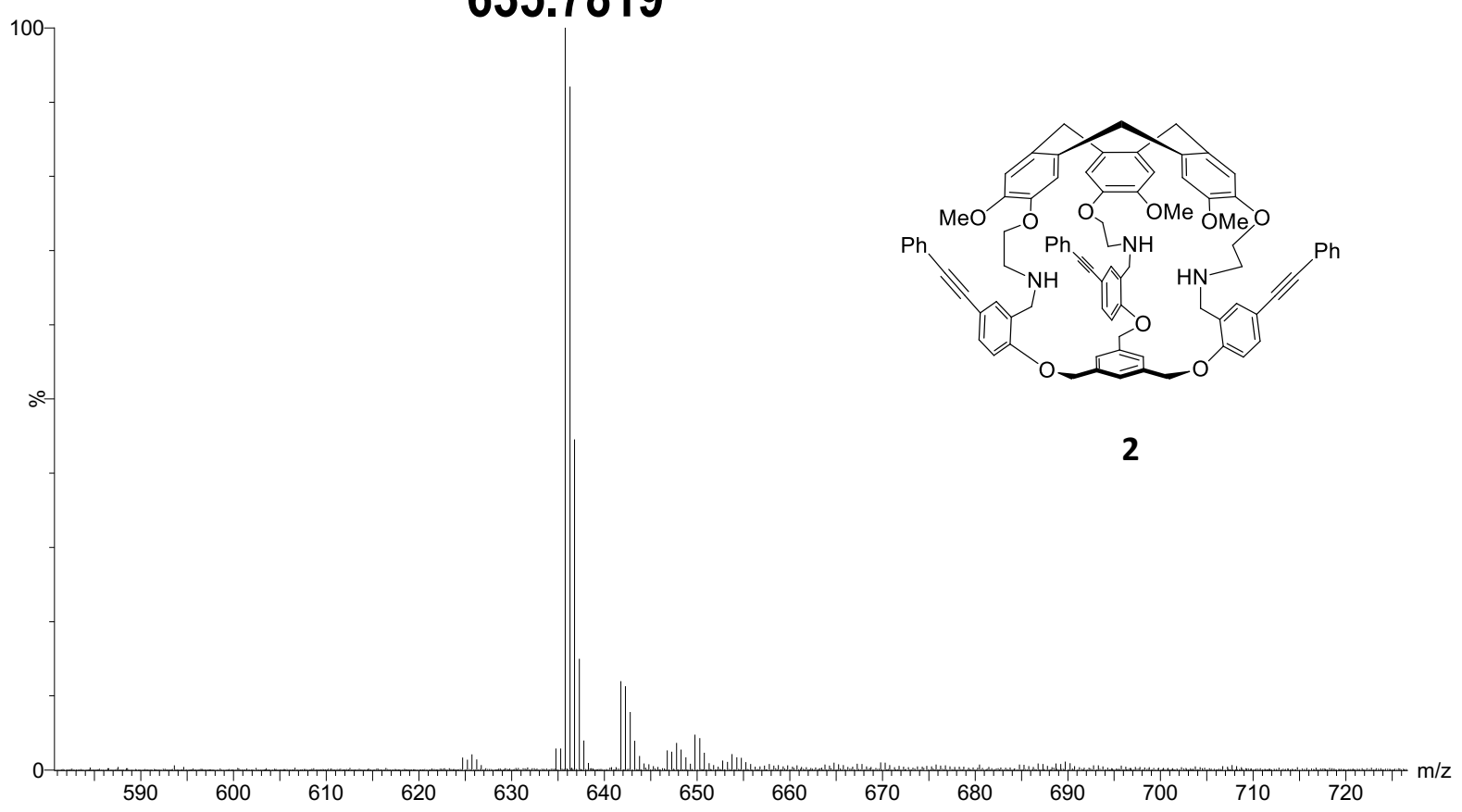

Figure S19. HRMS Spectrum of $[2+2 \mathrm{H}]^{2+}$. Expected peak is observed at 635.7819 .

\subsection{3}

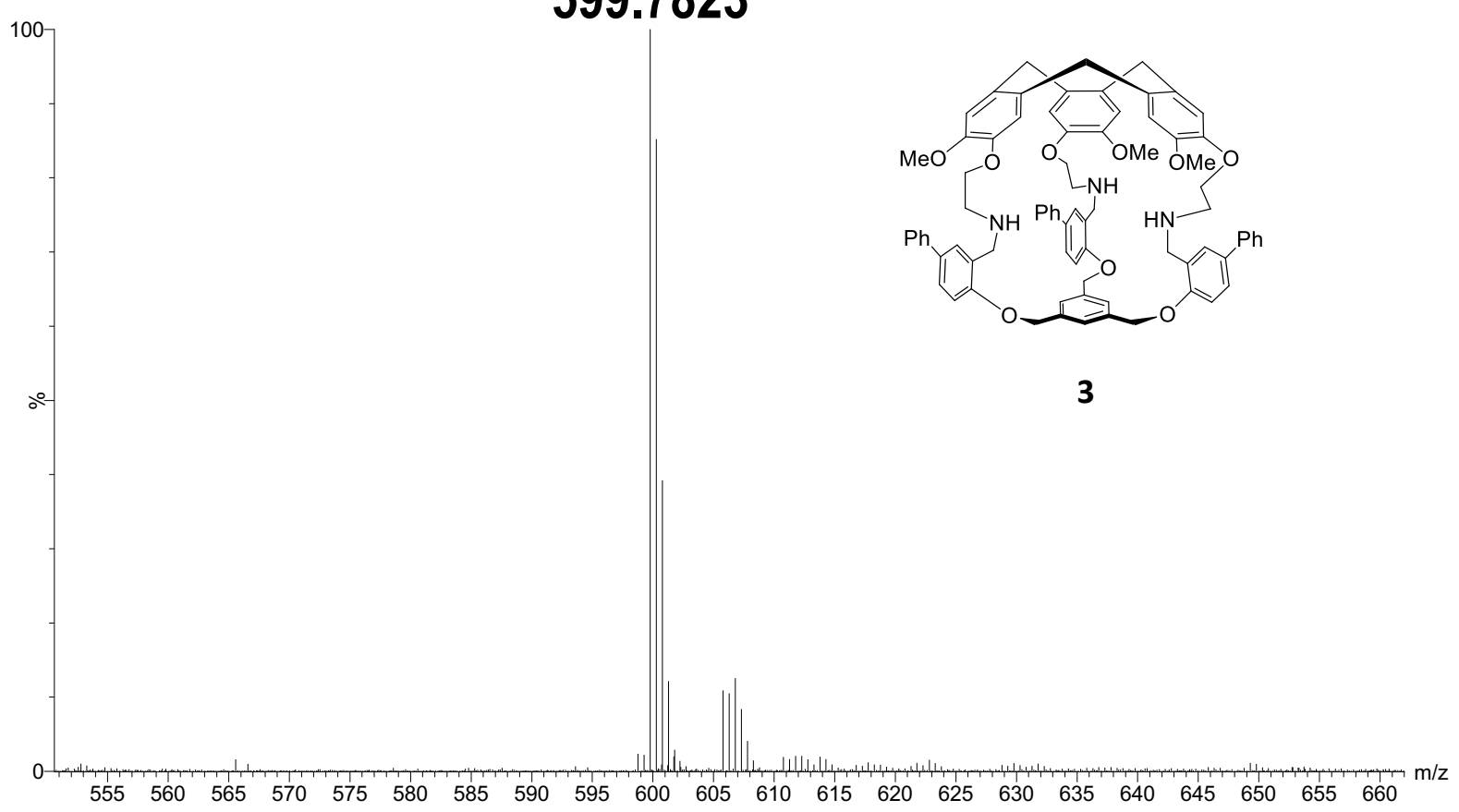

Figure S20. Spectrum of $[3+2 \mathrm{H}]^{2+}$. Expected peak is observed at 599.7823 . 


\section{Photophysical studies}

\subsection{Spectra and properties}

\section{Hemicryptophane 2}

Solution in DMSO $+2 \% \mathrm{H}_{2} \mathrm{O}, \mathrm{C}=3.2 \mu \mathrm{M}$.

Absorption: $\lambda_{\max }=294 \mathrm{~nm}, \varepsilon=9.4 \times 10^{4} \mathrm{~L} \cdot \mathrm{mol}^{-1} \cdot \mathrm{cm}^{-1}$.

Emission: $\lambda_{\text {exc }}=290 \mathrm{~nm}, \lambda_{\max }=352 \mathrm{~nm}$, quantum yield: $5.4 \%$.

\section{Hemicryptophane 3}

Solution in DMSO $+2 \% \mathrm{H}_{2} \mathrm{O}, \mathrm{C}=4.7 \mu \mathrm{M}$.

Absorption: $\lambda_{\max }=274 \mathrm{~nm}, \varepsilon=5.1 \times 10^{4} \mathrm{~L} \cdot \mathrm{mol}^{-1} \cdot \mathrm{cm}^{-1}$.

Emission: $\lambda_{\text {exc }}=290 \mathrm{~nm}, \lambda_{\max }=334 \mathrm{~nm}$, quantum yield: $7.5 \%$.

(a)

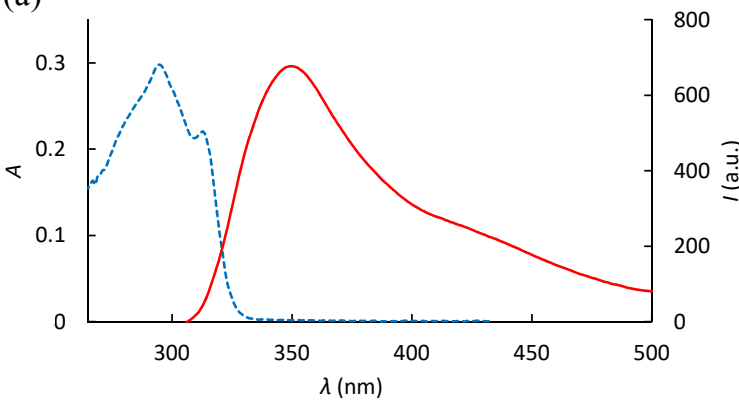

(b)

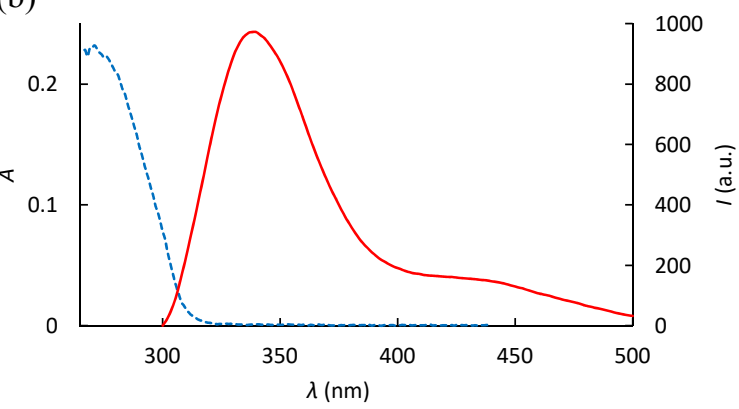

Figure S21. Absorption (blue, dotted line) and emission (red, solid line, excitation at $290 \mathrm{~nm}$ ) spectra in DMSO $+2 \% \mathrm{H}_{2} \mathrm{O}$ at $298 \mathrm{~K}$ of (a) a $3.2 \mu \mathrm{M}$ solution of hemicryptophane 2 and (b) a $4.7 \mu \mathrm{M}$ solution of hemicryptophane 3.

\subsection{Fluorescence titration}

\subsubsection{Titration curves}

\subsubsection{Acetylcholine}

(a)

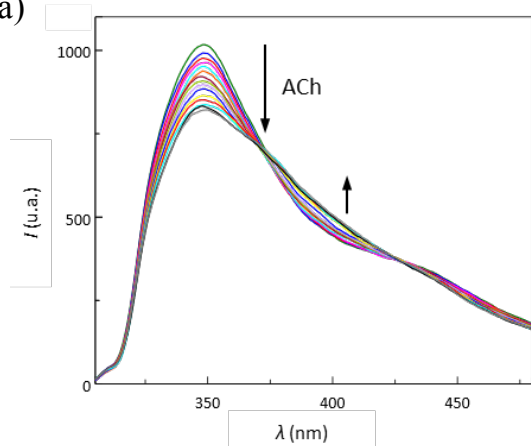

(b)

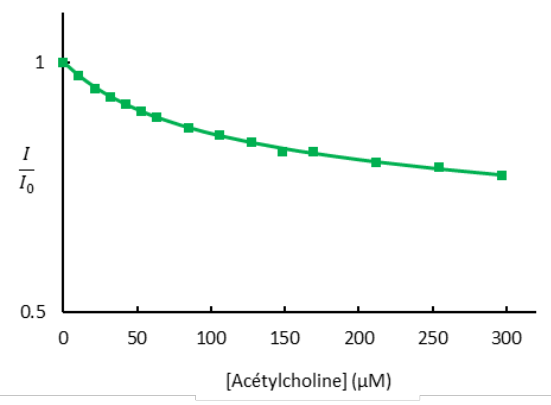

Figure S22. (a) Fluorescence titration of a $5.0 \mu \mathrm{M}$ solution of hemicryptophane 2 with acetylcholine (counterion $\mathrm{Cl}^{-}$) excited at $290 \mathrm{~nm}$ in DMSO $+2 \% \mathrm{H}_{2} \mathrm{O}$. (b) Relative fluorescence intensity (at $348 \mathrm{~nm}$ ) as a function of the concentration of acetylcholine. 

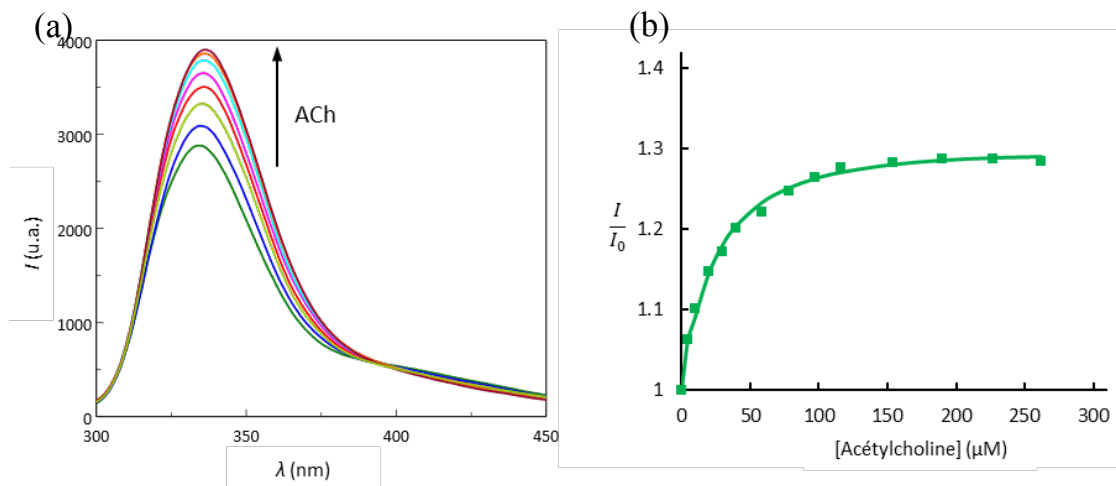

Figure S23. (a) Fluorescence titration of a $5.0 \mu \mathrm{M}$ solution of hemicryptophane 3 with acetylcholine (counterion $\mathrm{Cl}^{-}$) excited at $290 \mathrm{~nm}$ in DMSO $+2 \% \mathrm{H}_{2} \mathrm{O}$. (b) Relative fluorescence intensity (at $338 \mathrm{~nm}$ ) as a function of the concentration of acetylcholine.

\subsubsection{Choline}

(a)

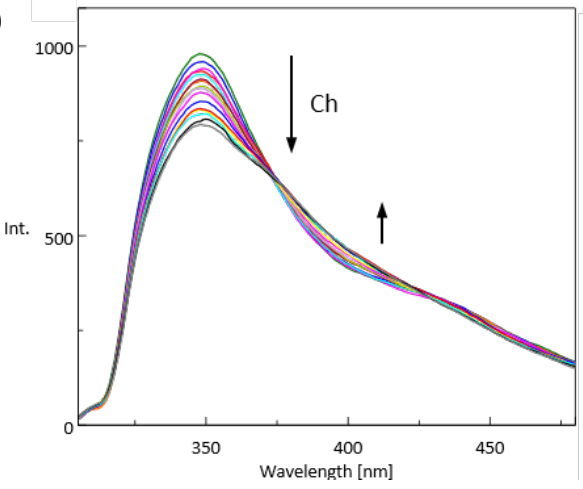

(b)

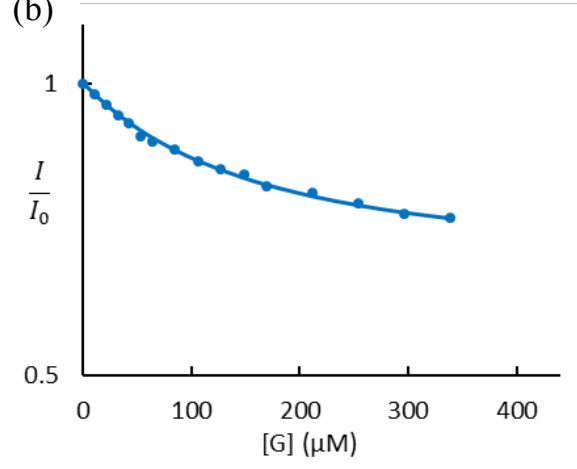

Figure S24. (a) Fluorescence titration of a $5.0 \mu \mathrm{M}$ solution of hemicryptophane 2 with choline (counterion $\mathrm{Cl}^{-}$) excited at $290 \mathrm{~nm}$ in DMSO $+2 \% \mathrm{H}_{2} \mathrm{O}$. (b) Relative fluorescence intensity (at $348 \mathrm{~nm}$ ) as a function of the concentration of choline.

(a) 50 (a)

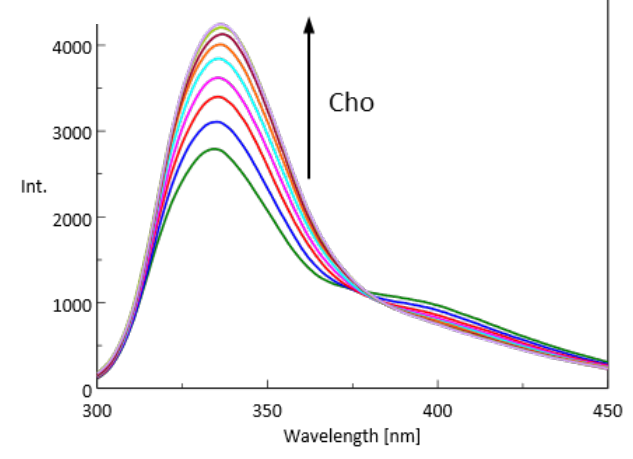

(b) 1. (b)

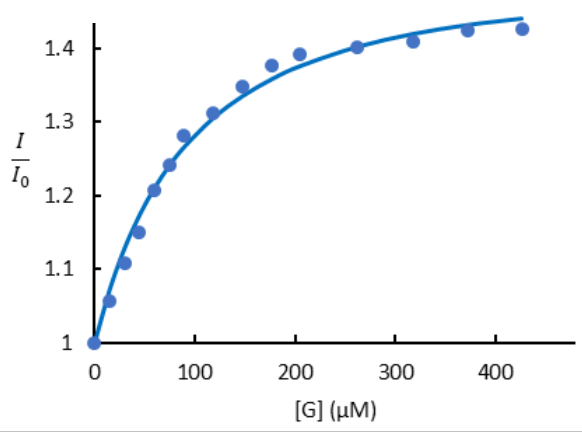

Figure S25. (a) Fluorescence titration of a $5.0 \mu \mathrm{M}$ solution of hemicryptophane 3 with choline (counterion $\mathrm{Cl}^{-}$) excited at $290 \mathrm{~nm}$ in DMSO $+2 \% \mathrm{H}_{2} \mathrm{O}$. (b) Relative fluorescence intensity (at $338 \mathrm{~nm}$ ) as a function of the concentration of choline. 


\subsubsection{Fit parameters}

\begin{tabular}{|c|c|c|c|c|}
\hline Guest & Host & $K_{a}\left(\mathrm{~L} . \mathrm{mol}^{-1}\right)$ & cov & RMS \\
\hline \multirow{3}{*}{ Acetylcholine } & $\mathbf{1}$ & $2.4 \times 10^{4} \pm 2.0 \%$ & $6.91 \times 10^{-4}$ & $2.46 \times 10^{-2}$ \\
\cline { 2 - 5 } & $\mathbf{2}$ & $1.2 \times 10^{4} \pm 2.1 \%$ & $4.69 \times 10^{-4}$ & $2.39 \times 10^{-3}$ \\
\cline { 2 - 5 } & $\mathbf{3}$ & $4.4 \times 10^{4} \pm 4.4 \%$ & $3.88 \times 10^{-3}$ & $5.33 \times 10^{-3}$ \\
\hline \multirow{3}{*}{ Choline } & $\mathbf{1}$ & $5.9 \times 10^{3} \pm 2.3 \%$ & $7.78 \times 10^{-4}$ & $2.73 \times 10^{-2}$ \\
\cline { 2 - 5 } & $\mathbf{2}$ & $4.3 \times 10^{3} \pm 2.2 \%$ & $1.41 \times 10^{-3}$ & $3.97 \times 10^{-3}$ \\
\cline { 2 - 5 } & $\mathbf{3}$ & $1.0 \times 10^{4} \pm 4.8 \%$ & $3.71 \times 10^{-3}$ & $1.34 \times 10^{-2}$ \\
\hline
\end{tabular}

Table S1. $K_{\mathrm{a}}$, covariance and RMS obtained from fits of the titration curves. 


\section{3. ${ }^{1} \mathrm{H}$ NMR Titrations}

\subsection{Titration curves}

\subsubsection{Hemicryptophane 2}

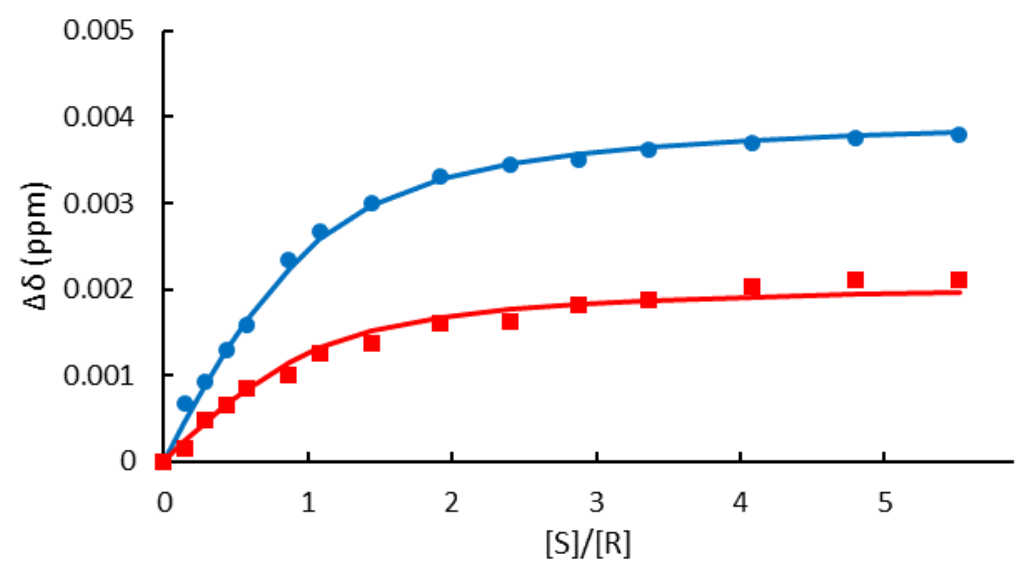

Figure S26. Titration curve of host 2 with acetylcholine. The chemical induced shifts $\Delta \delta$ of host's protons at 7.32 $\mathrm{ppm}(\bigcirc)$ and $6.75 \mathrm{ppm}(\square)$ were measured and plotted as a function of the ratio $[\mathrm{S}] /[\mathrm{R}]$ (dots). Curves were fitted with the bindfit program (lines).

\subsubsection{Hemicryptophane 3}

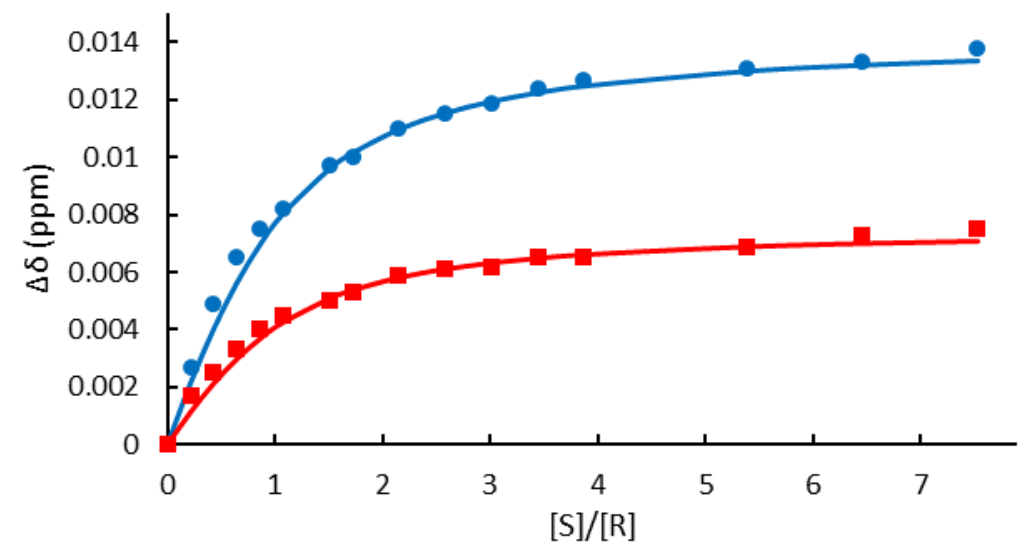

Figure S27. Titration curve of host 3 with acetylcholine. The chemical induced shifts $\Delta \delta$ of host's protons at 7.42 ppm (๑) and $6.77 \mathrm{ppm}(\square)$ were measured and plotted as a function of the ratio $[\mathrm{S}] /[\mathrm{R}]$ (dots). Curves were fitted with the bindfit program (lines). 


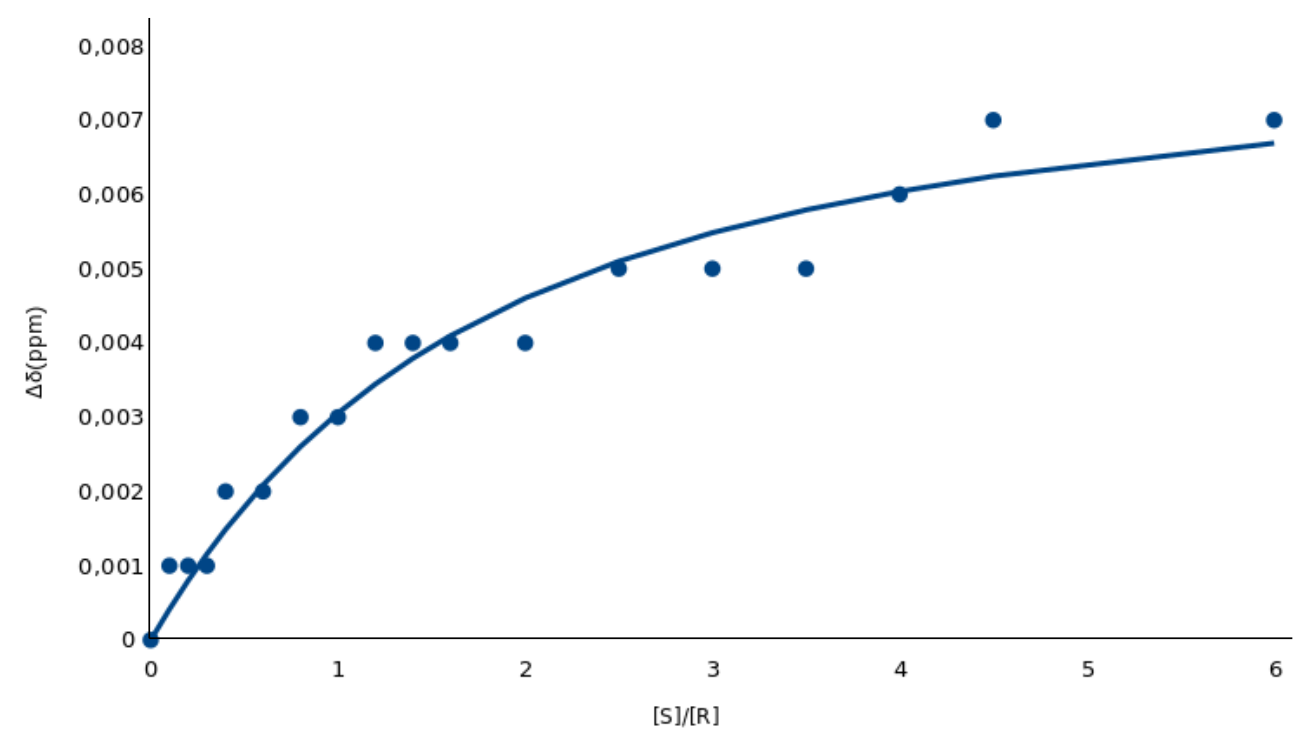

Figure S28. Titration curve of host 2 with choline. The chemical induced shifts $\Delta \delta$ of host's protons at $4.69 \mathrm{ppm}$ $(\bigcirc)$ were measured and plotted as a function of the ratio $[\mathrm{S}] /[\mathrm{R}]$ (dots). The curve was fitted with the bindfit program (lines).

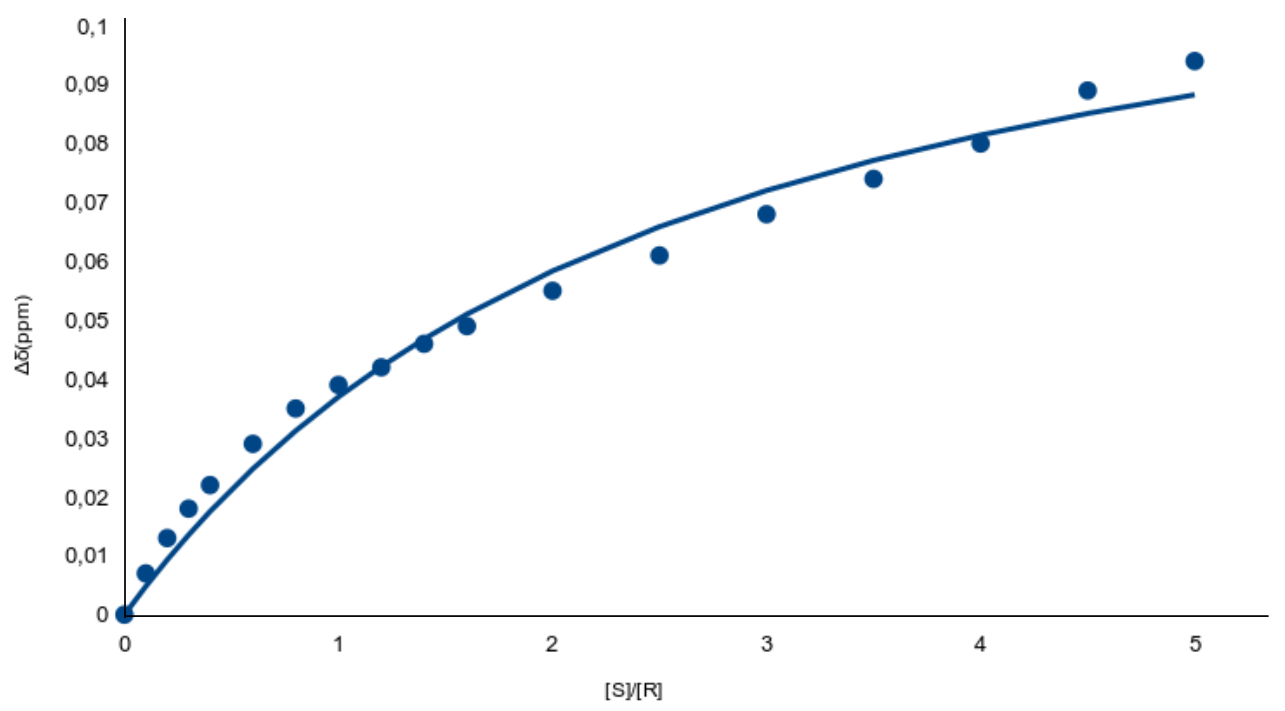

Figure S29. Titration curve of host 3 choline. The chemical induced shifts $\Delta \delta$ of host's protons at $7.40 \mathrm{ppm}(\bullet)$ were measured and plotted as a function of the ratio $[\mathrm{S}] /[\mathrm{R}]$ (dots). The curve was fitted with the bindfit program (lines). 


\section{2. ${ }^{1} \mathrm{H}$ NMR spectra}

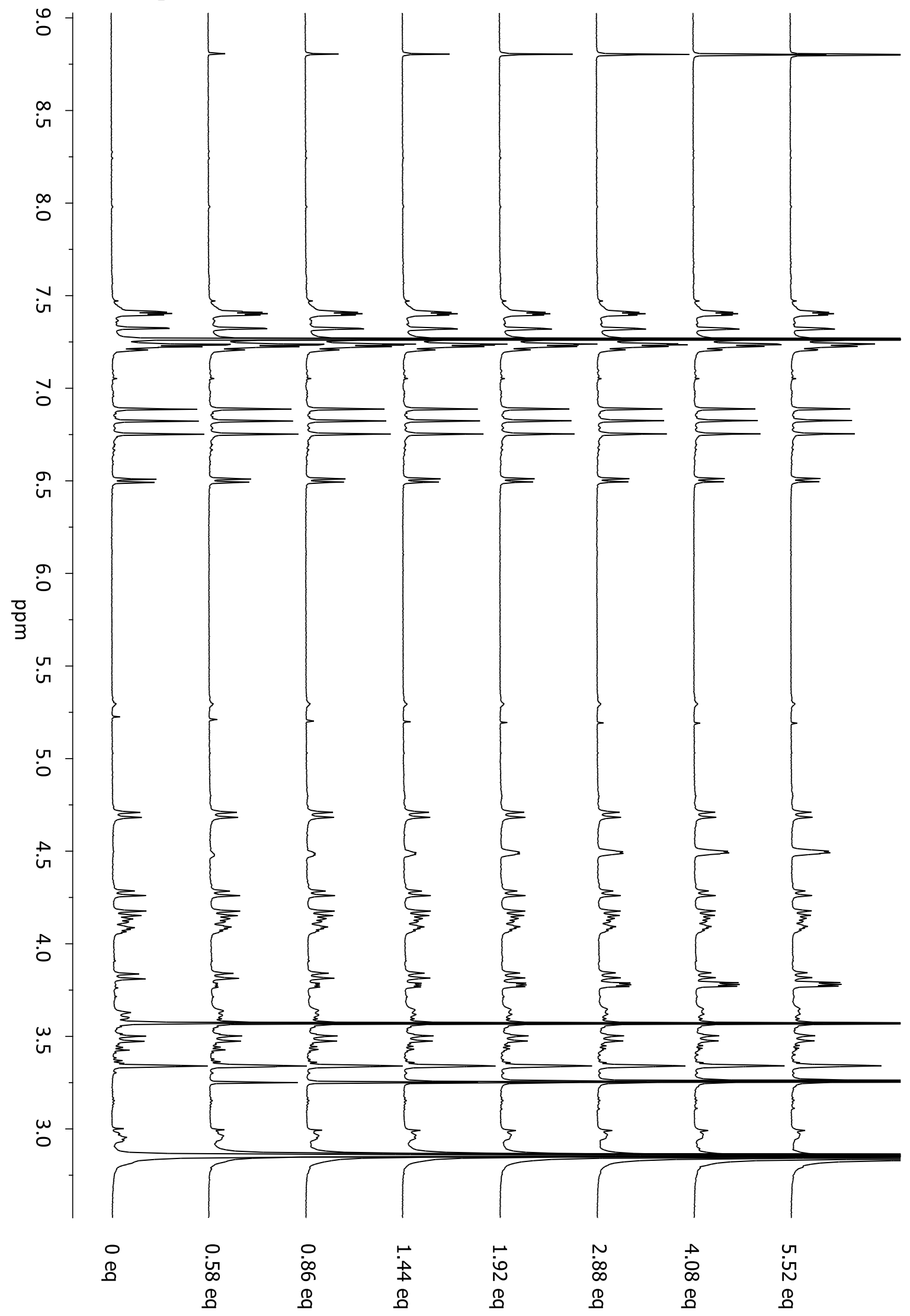

Figure S30. ${ }^{1} \mathrm{H}$ NMR spectra of $2\left(1.0 \mathrm{mM}\right.$ in $\left.\mathrm{CDCl}_{3} / \mathrm{MeOD} 95 / 5\right)$ for several additions of a concentrated solution of acetylcholine picrate (5.0 $\mathrm{mM}$ in the same solvents). 


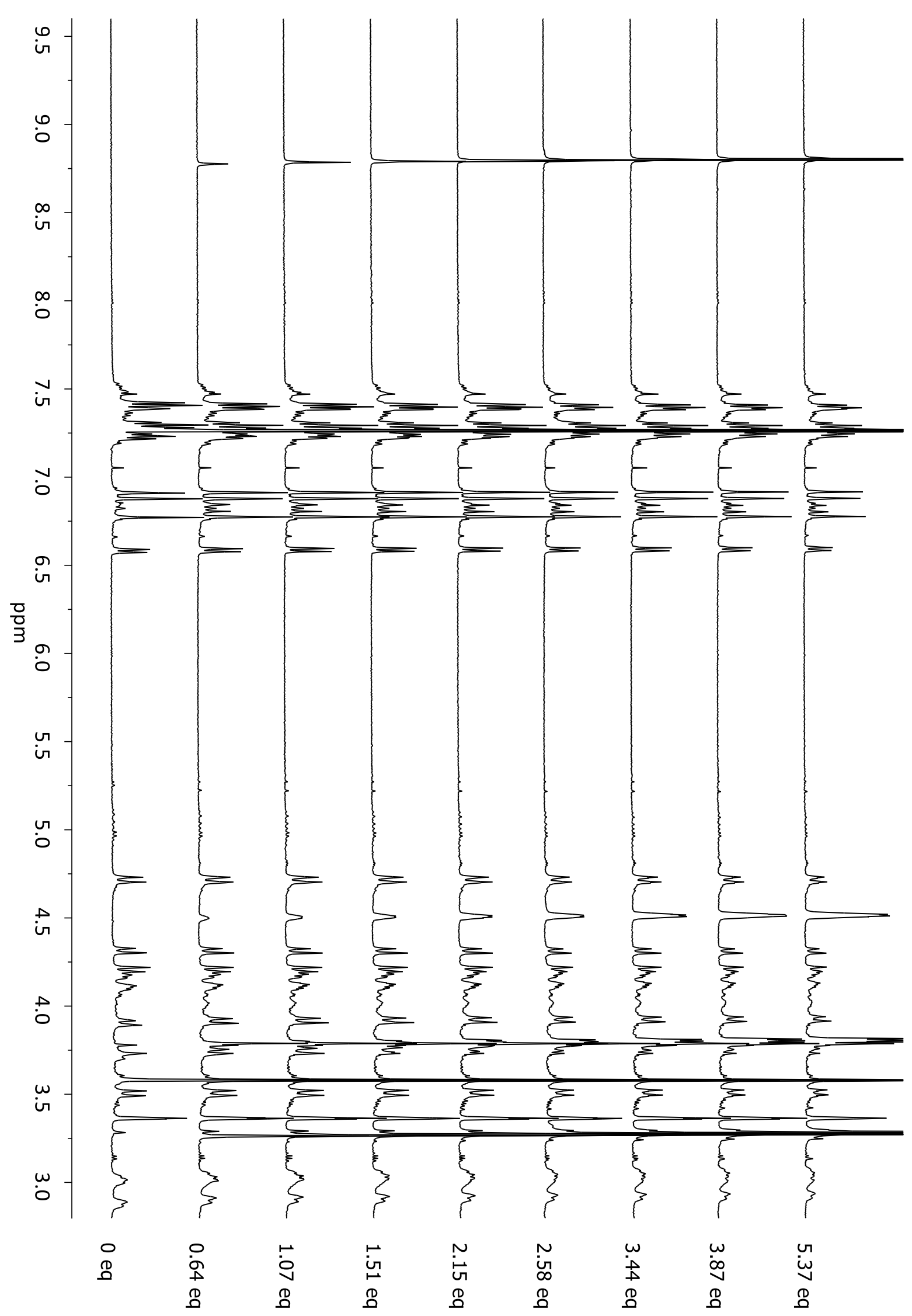

Figure $\mathrm{S} 31{ }^{1} \mathrm{H}$ NMR spectra of $\mathbf{3}\left(1.0 \mathrm{mM}\right.$ in $\left.\mathrm{CDCl}_{3} / \mathrm{MeOD} 95 / 5\right)$ for several additions of a concentrated solution of acetylcholine picrate $(5.0 \mathrm{mM}$ in the same solvents). 

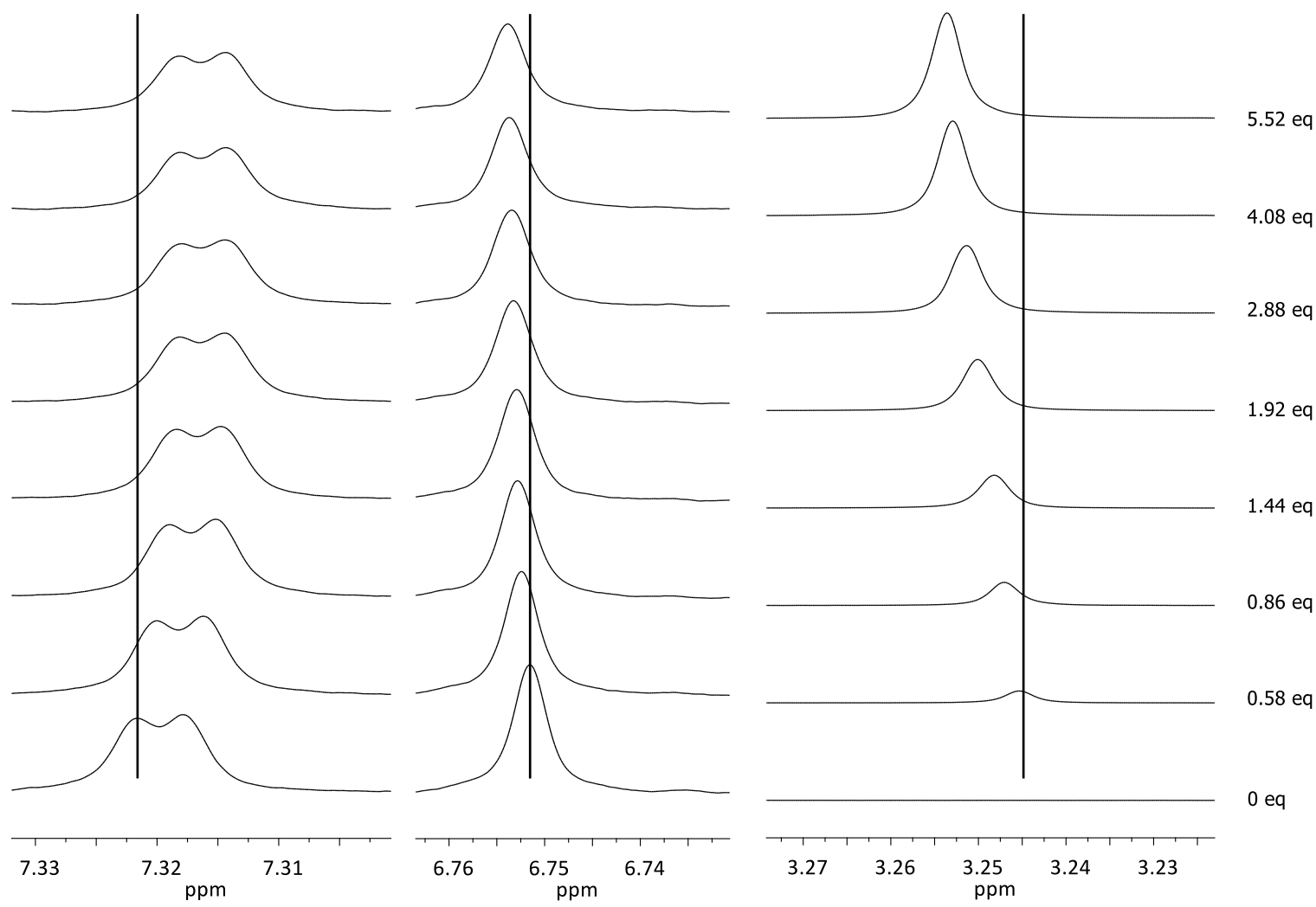

Figure S32. Zooms on several signals on ${ }^{1} \mathrm{H}$ NMR spectra of $\mathbf{2}$ for the titration with ACh.
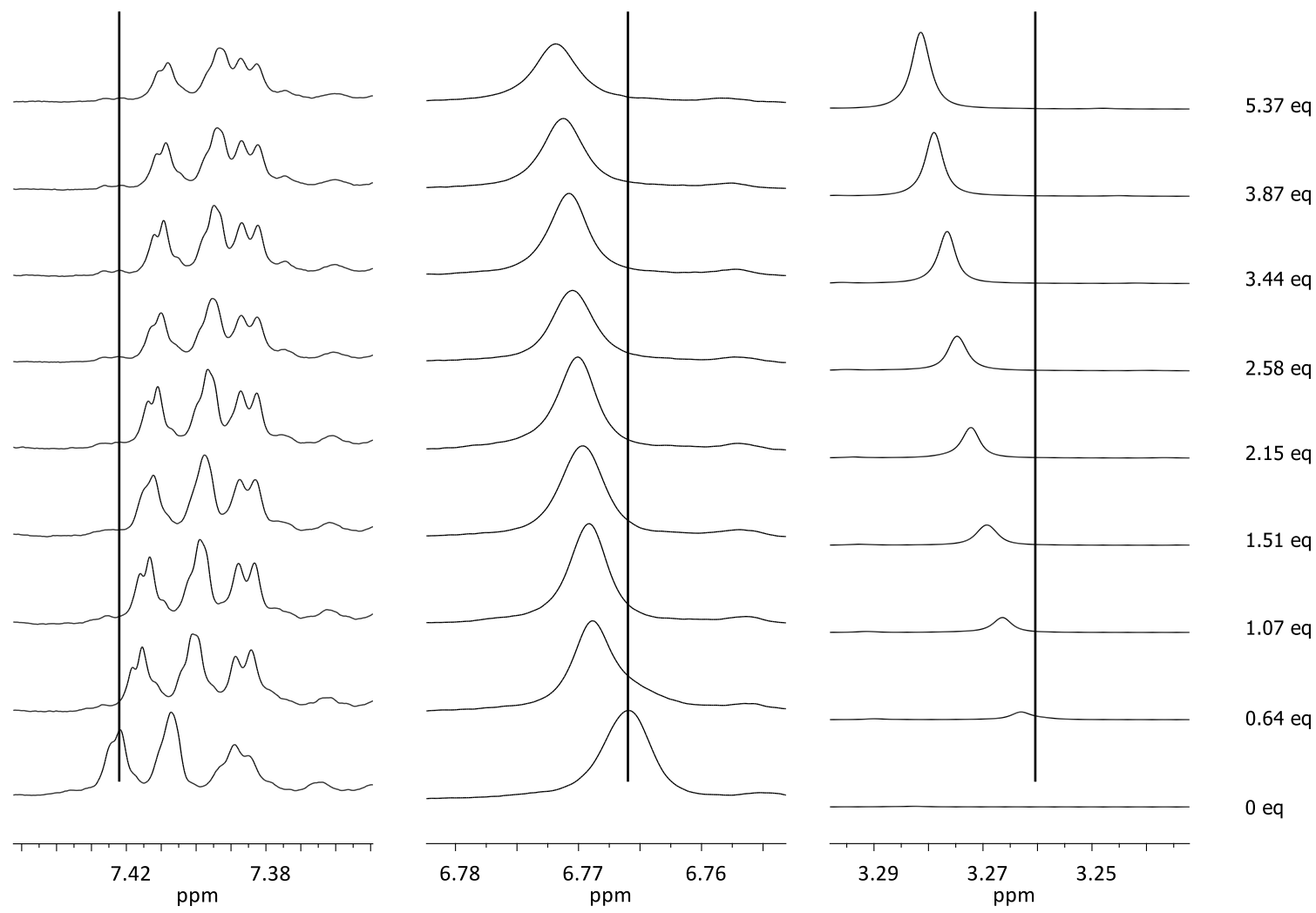

Figure S33. Zooms on several signals on ${ }^{1} \mathrm{H}$ NMR spectra of $\mathbf{3}$ for the titration with ACh. 


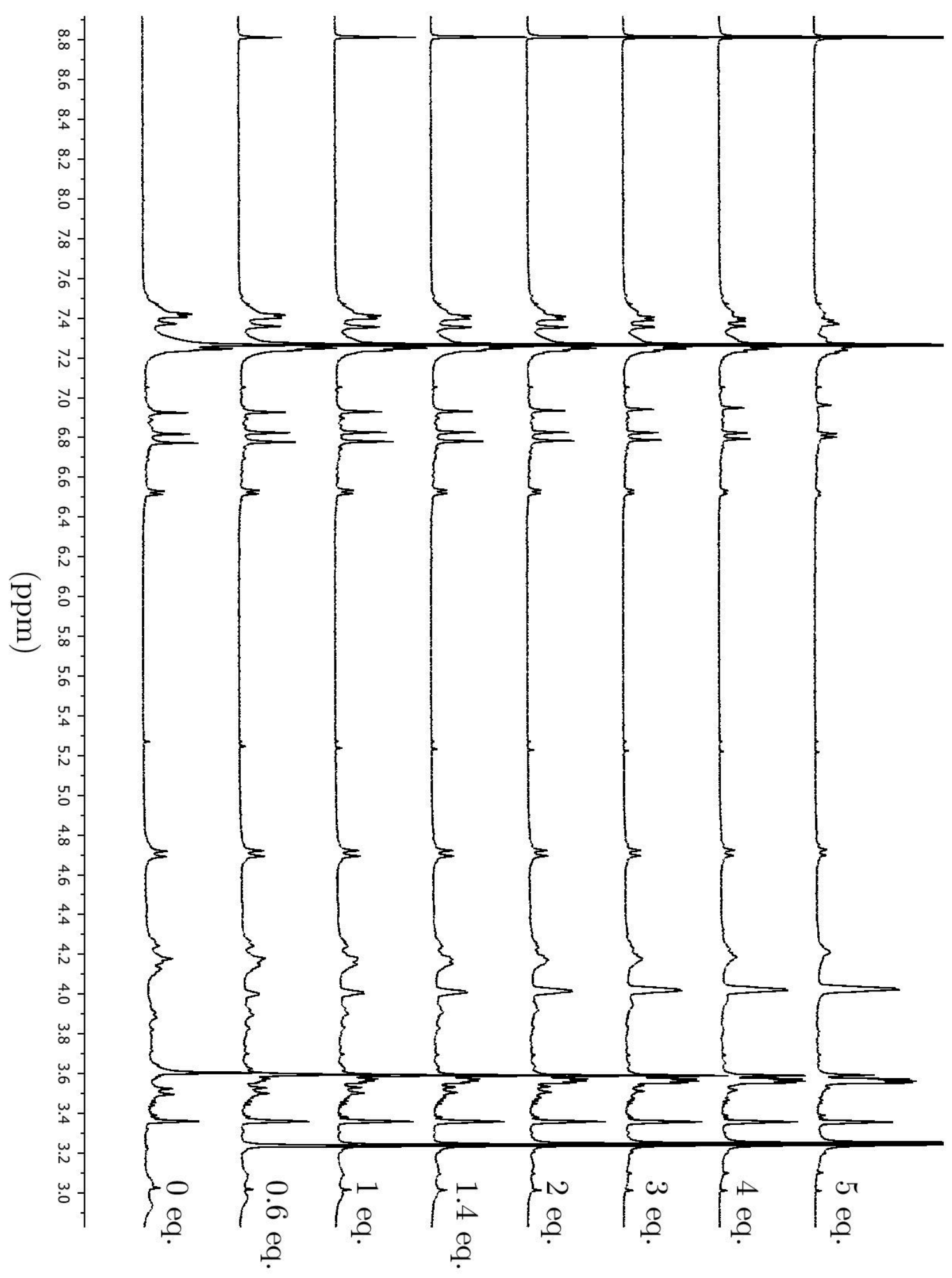

Figure S34. ${ }^{1} \mathrm{H}$ NMR spectra of $2\left(1.0 \mathrm{mM}\right.$ in $\left.\mathrm{CDCl}_{3} / \mathrm{MeOD} 95 / 5\right)$ for several additions of a concentrated solution of choline picrate (5.0 $\mathrm{mM}$ in the same solvents). 


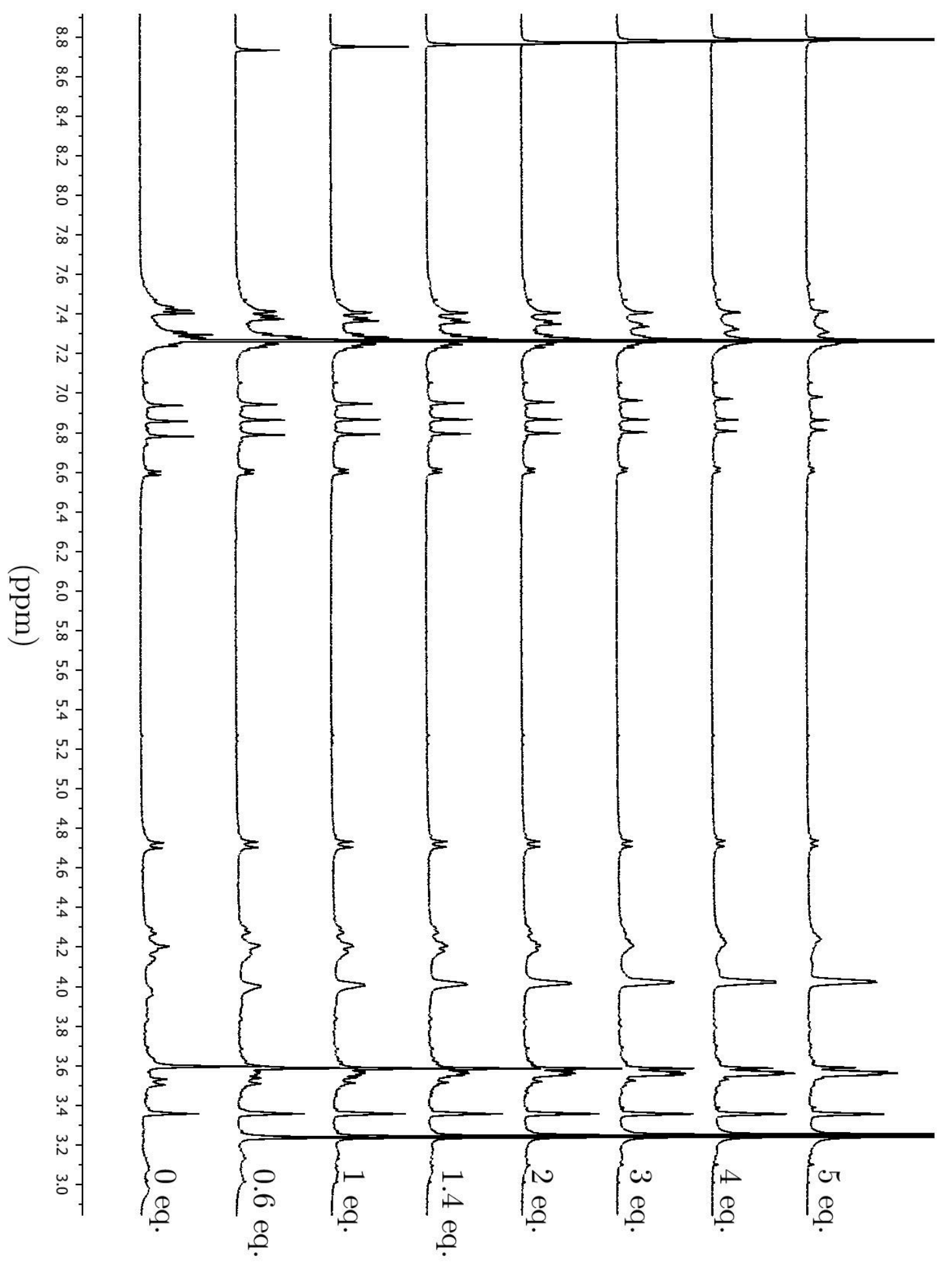

Figure S35. ${ }^{1} \mathrm{H}$ NMR spectra of $\mathbf{3}\left(1.0 \mathrm{mM}\right.$ in $\left.\mathrm{CDCl}_{3} / \mathrm{MeOD} 95 / 5\right)$ for several additions of a concentrated solution of choline picrate $(5.0 \mathrm{mM}$ in the same solvents). 

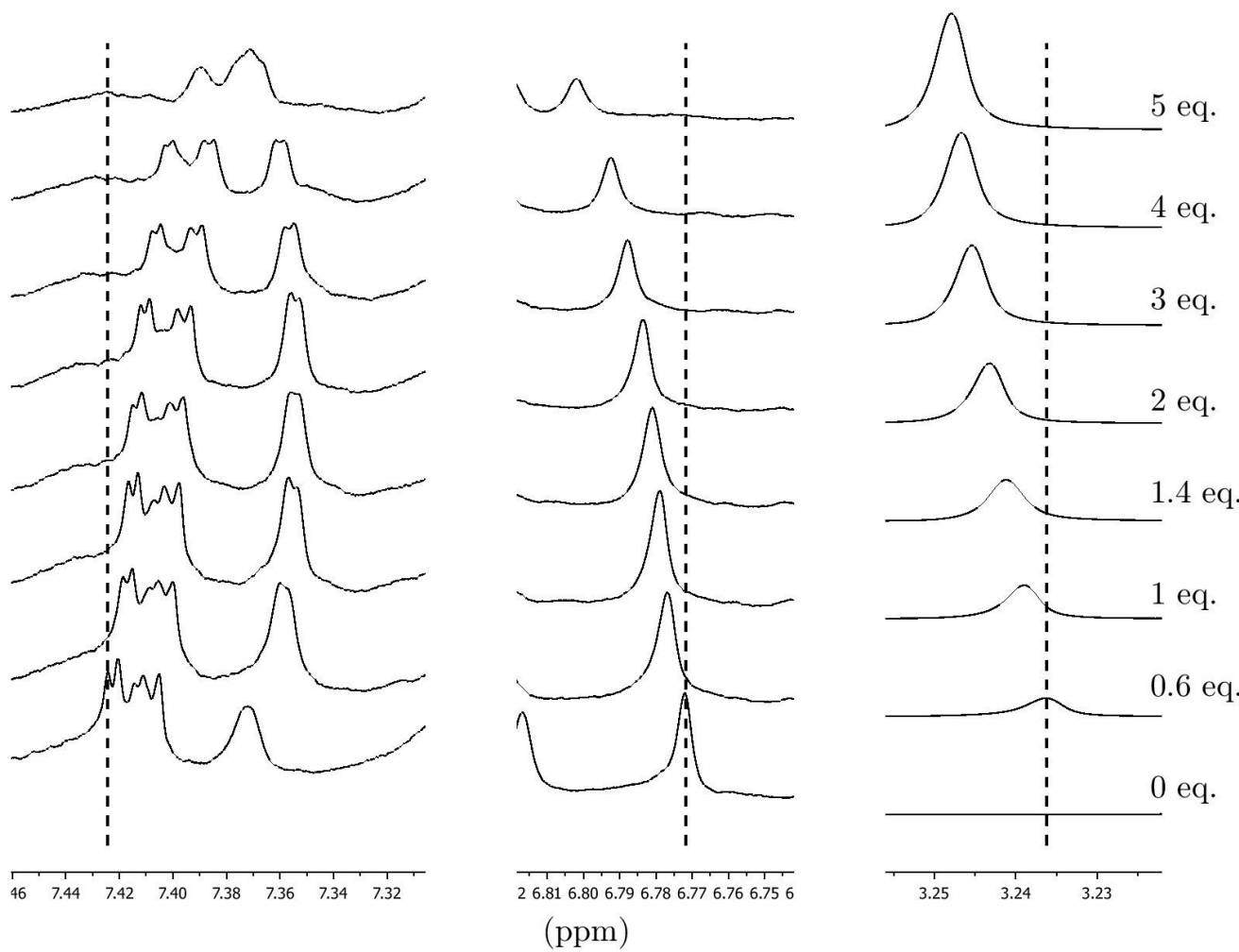

Figure S36. Zooms on several signals on ${ }^{1} \mathrm{H}$ NMR spectra of 2 for the titration with Ch.
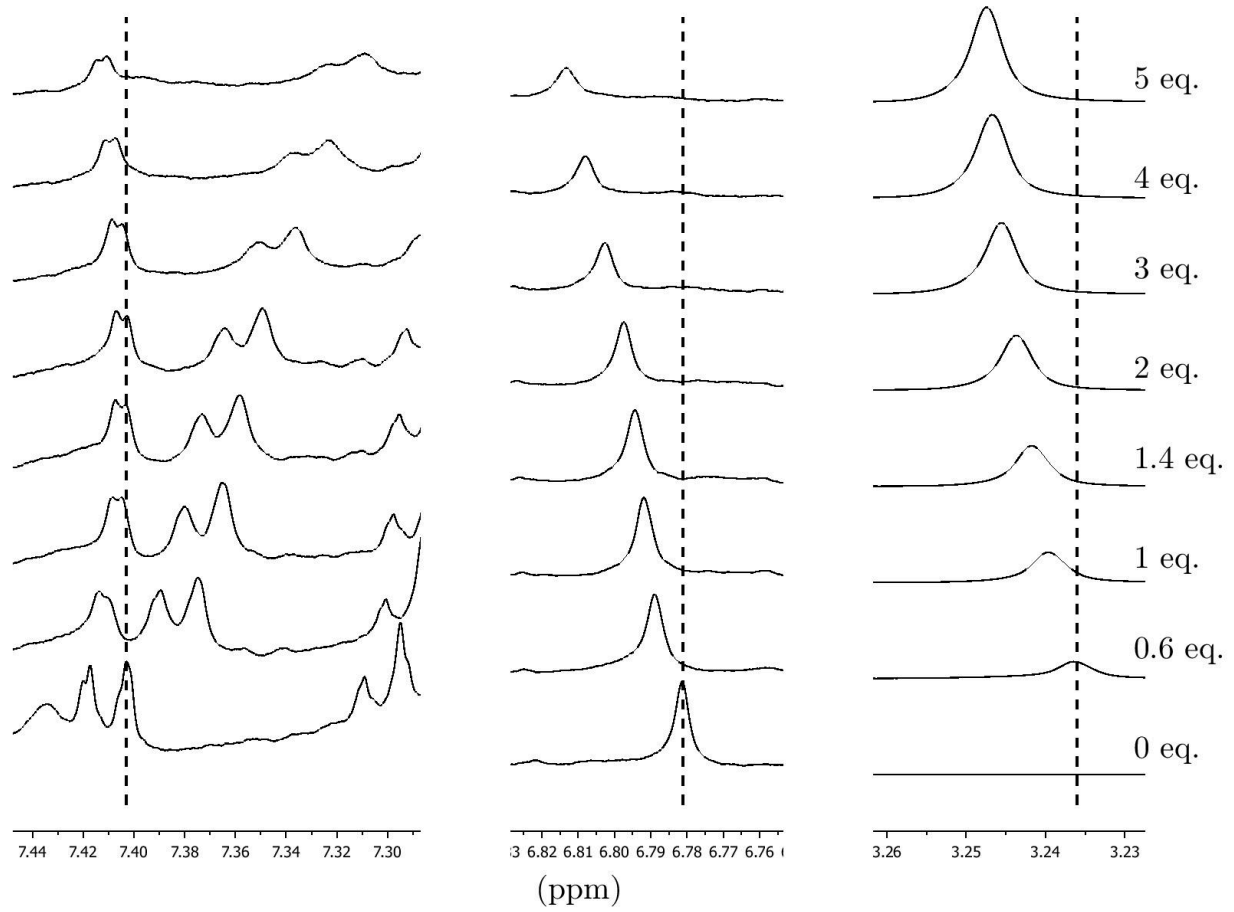

$\begin{array}{lllllllll}7.44 & 7.42 & 7.40 & 7.38 & 7.36 & 7.34 & 7.32 & 7.30\end{array}$

$$
\text { (ppm) }
$$

Figure S37. Zooms on several signals on ${ }^{1} \mathrm{H}$ NMR spectra of $\mathbf{3}$ for the titration with $\mathrm{Ch}$ 


\subsection{Fit parameters}

\begin{tabular}{|c|c|c|c|c|}
\hline Host & Guest & $K_{\mathrm{a}}\left({\left.\mathrm{L} . \mathrm{mol}^{-1}\right)}^{-1}\right.$ & cov & RMS (ppm) \\
\hline $\mathbf{2}$ & ACh & $4.7 \times 10^{3} \pm 6.7 \%$ & $5.48 \times 10^{-3}$ & $8.74 \times 10^{-5}$ \\
\hline $\mathbf{3}$ & ACh & $6.2 \times 10^{3} \pm 7.1 \%$ & $5.56 \times 10^{-3}$ & $2.92 \times 10^{-4}$ \\
\hline $\mathbf{2}$ & Ch & $6.49 \times 10^{2} \pm 12.1 \%$ & $4.07 \times 10^{-2}$ & $4.15 \times 10^{-4}$ \\
\hline $\mathbf{3}$ & Ch & $2.9 \times 10^{2} \pm 5.6 \%$ & $1.48 \times 10^{-2}$ & $3.42 \times 10^{-3}$ \\
\hline
\end{tabular}

Table S2. $K_{\mathrm{a}}$, covariance and RMS obtained from fits of the titration curves for acetylcholine and choline. ${ }^{1}$

\section{DFT calculations}

Compound 2.

Cartesian coordinates

197

$\mathrm{E}(\mathrm{RB} 3 \mathrm{LYP})=-4568.41726710$

$\begin{array}{lccc}\mathrm{C} & -6.002217 & 12.183761 & -2.397987 \\ \mathrm{C} & -4.512934 & 2.014177 & 0.003130 \\ \mathrm{C} & -8.784899 & -8.775218 & 0.147336 \\ \mathrm{C} & -0.237958 & 1.568011 & -0.098725 \\ \mathrm{C} & -1.686331 & 1.646506 & 0.362239 \\ \mathrm{C} & -9.911674 & -10.266637 & -1.930055 \\ \mathrm{C} & 0.074889 & -0.898823 & -0.426414 \\ \mathrm{C} & -9.823275 & -9.664637 & 0.410236 \\ \mathrm{C} & -5.281550 & 13.152012 & -1.693460 \\ \mathrm{C} & 13.156352 & -3.627285 & -1.355007 \\ \mathrm{C} & -3.497783 & 11.559670 & -1.323539 \\ \mathrm{C} & 11.299693 & -2.397773 & -2.328691 \\ \mathrm{C} & 13.589864 & -1.805091 & -2.885481 \\ \mathrm{C} & 1.999838 & 0.586576 & -0.181522 \\ \mathrm{C} & -3.217621 & 8.152882 & -2.361477 \\ \mathrm{C} & -4.216772 & 10.576092 & -2.032719 \\ \mathrm{C} & 0.577965 & 0.113452 & 1.764852 \\ \mathrm{C} & -3.830448 & 0.745767 & -0.439754 \\ \mathrm{C} & -10.390182 & -10.412806 & -0.625158 \\ \mathrm{C} & -4.029946 & 12.835528 & -1.158093 \\ \mathrm{C} & 0.427741 & 0.732048 & -4.001376 \\ \mathrm{C} & 14.062008 & -2.821718 & -2.050743 \\ \mathrm{C} & -8.873611 & -9.379728 & -2.202606 \\ \mathrm{C} & -0.970238 & 0.747013 & -3.879004 \\ \mathrm{C} & -1.659690 & -0.466185 & -3.855590 \\ \mathrm{C} & -3.676724 & 9.268924 & -2.207744 \\ \mathrm{C} & -0.982306 & -1.687381 & -3.988949 \\ \mathrm{C} & -6.325037 & -6.934624 & -1.673136\end{array}$




\begin{tabular}{|c|c|c|c|}
\hline $\mathrm{C}$ & -7.232055 & -7.710919 & -1.439165 \\
\hline $\mathrm{C}$ & 8.701852 & -1.997259 & -2.593779 \\
\hline $\mathrm{C}$ & 9.897999 & -2.182402 & -2.470123 \\
\hline $\mathrm{C}$ & 0.408504 & -1.682114 & -4.105443 \\
\hline $\mathrm{C}$ & 1.125393 & -0.475198 & -4.097869 \\
\hline C & 2.631627 & -0.477235 & -4.198157 \\
\hline $\mathrm{C}$ & -1.774071 & -2.974712 & -4.004527 \\
\hline $\mathrm{C}$ & -1.744547 & 2.043122 & -3.835795 \\
\hline $\mathrm{C}$ & 11.786130 & -3.420681 & -1.489363 \\
\hline $\mathrm{C}$ & 0.479603 & 4.934335 & -1.566212 \\
\hline $\mathrm{C}$ & -5.478509 & 10.905182 & -2.568677 \\
\hline $\mathrm{C}$ & 4.055797 & -3.134438 & -1.214595 \\
\hline $\mathrm{C}$ & -3.001612 & -3.635825 & 0.006674 \\
\hline $\mathrm{C}$ & 4.703801 & -3.294425 & 2.000149 \\
\hline $\mathrm{C}$ & 1.578081 & 5.318023 & 2.049453 \\
\hline $\mathrm{C}$ & 1.695497 & 4.907468 & 0.578330 \\
\hline $\mathrm{C}$ & 4.488263 & -2.125810 & 1.028275 \\
\hline $\mathrm{C}$ & -4.392600 & -1.387371 & 2.729665 \\
\hline $\mathrm{C}$ & -3.355693 & -2.151195 & 1.878174 \\
\hline $\mathrm{C}$ & -3.393411 & 5.865234 & -3.260231 \\
\hline $\mathrm{C}$ & -2.684806 & 6.846101 & -2.543877 \\
\hline $\mathrm{C}$ & -2.868510 & 4.587545 & -3.431255 \\
\hline $\mathrm{C}$ & -1.617450 & 4.254212 & -2.891946 \\
\hline $\mathrm{C}$ & -0.879179 & 5.221580 & -2.176561 \\
\hline $\mathrm{C}$ & -1.428701 & 6.493582 & -2.014325 \\
\hline $\mathrm{C}$ & 6.798406 & -0.854036 & -3.665408 \\
\hline $\mathrm{C}$ & 7.303246 & -1.779560 & -2.737475 \\
\hline $\mathrm{C}$ & 5.426285 & -0.655289 & -3.802616 \\
\hline $\mathrm{C}$ & 4.521836 & -1.367011 & -3.005525 \\
\hline $\mathrm{C}$ & 4.997327 & -2.307536 & -2.063128 \\
\hline $\mathrm{C}$ & 6.373541 & -2.497611 & -1.956675 \\
\hline $\mathrm{C}$ & -5.264310 & -6.025548 & -1.946632 \\
\hline $\mathrm{C}$ & -4.769395 & -5.857449 & -3.252003 \\
\hline $\mathrm{C}$ & -4.669225 & -5.274650 & -0.914104 \\
\hline $\mathrm{C}$ & -3.630915 & -4.377996 & -1.153085 \\
\hline $\mathrm{C}$ & -3.176247 & -4.204692 & -2.477478 \\
\hline $\mathrm{C}$ & -3.737802 & -4.960649 & -3.515028 \\
\hline $\mathrm{C}$ & 2.135410 & -1.326056 & 4.883460 \\
\hline $\mathrm{C}$ & 3.028922 & -2.004405 & 4.029526 \\
\hline $\mathrm{C}$ & 2.651335 & -3.117497 & 3.286153 \\
\hline $\mathrm{C}$ & 1.327891 & -3.606313 & 3.412352 \\
\hline $\mathrm{C}$ & 0.447672 & -2.946354 & 4.264476 \\
\hline $\mathrm{C}$ & 0.819311 & -1.799666 & 4.994823 \\
\hline $\mathrm{C}$ & 1.533409 & 2.126549 & 5.066623 \\
\hline $\mathrm{C}$ & 1.575047 & 3.330332 & 4.338207 \\
\hline $\mathrm{C}$ & 2.592582 & 3.630659 & 3.437918 \\
\hline $\mathrm{C}$ & 3.638713 & 2.697648 & 3.254143 \\
\hline $\mathrm{C}$ & 3.621439 & 1.518629 & 3.999723 \\
\hline $\mathrm{C}$ & 2.582346 & 1.207223 & 4.897521 \\
\hline $\mathrm{C}$ & 2.671066 & -0.118829 & 5.648176 \\
\hline
\end{tabular}




\begin{tabular}{|c|c|c|c|}
\hline $\mathrm{C}$ & -0.857552 & 1.290742 & 5.284601 \\
\hline $\mathrm{C}$ & -1.751997 & 2.180870 & 4.658251 \\
\hline $\mathrm{C}$ & -2.829325 & 1.743107 & 3.897940 \\
\hline $\mathrm{C}$ & -3.035276 & 0.350450 & 3.728997 \\
\hline $\mathrm{C}$ & -2.186950 & -0.527669 & 4.400010 \\
\hline $\mathrm{C}$ & -1.096633 & -0.086528 & 5.179194 \\
\hline $\mathrm{C}$ & -0.244620 & -1.147747 & 5.873342 \\
\hline $\mathrm{C}$ & 0.345600 & 1.897179 & 5.998474 \\
\hline $\mathrm{C}$ & 5.774769 & 2.247436 & 2.285217 \\
\hline $\mathrm{C}$ & -0.192515 & -5.381439 & 2.925155 \\
\hline $\mathrm{C}$ & -3.715505 & 3.947842 & 3.609511 \\
\hline $\mathrm{C}$ & -8.294673 & -8.620226 & -1.165417 \\
\hline $\mathrm{C}$ & 12.221455 & -1.591168 & -3.026761 \\
\hline $\mathrm{H}$ & 5.189439 & -4.137608 & 1.501858 \\
\hline $\mathrm{H}$ & 5.338289 & -2.996464 & 2.845212 \\
\hline $\mathrm{H}$ & 1.577134 & 6.406736 & 2.142235 \\
\hline $\mathrm{H}$ & 0.635264 & 4.943871 & 2.472933 \\
\hline $\mathrm{H}$ & 2.5 & 5.44 & 0.1 \\
\hline $\mathrm{H}$ & 1.956061 & 3.830391 & 0.5 \\
\hline $\mathrm{H}$ & 6533 & -1.842544 & 0.580536 \\
\hline $\mathrm{H}$ & 4.135109 & -1.252232 & 758 \\
\hline $\mathrm{H}$ & -4.553163 & -1.859605 & 5219 \\
\hline $\mathrm{H}$ & -5.351241 & -1.369329 & 2.207212 \\
\hline $\mathrm{H}$ & -11.200333 & -11.105809 & -0.41632 \\
\hline $\mathrm{H}$ & -2.599624 & -1.420429 & 1.531718 \\
\hline $\mathrm{H}$ & -2.8 & -2.882278 & 2.492901 \\
\hline $\mathrm{H}$ & 101 & -3.241638 & -1.259257 \\
\hline $\mathrm{H}$ & -0.864766 & 7.230959 & -1.4 \\
\hline $\mathrm{H}$ & -4.3 & 6.10 & 5779 \\
\hline $\mathrm{H}$ & 771 & -1.65 & 5772 \\
\hline $\mathrm{H}$ & -0.558553 & -3.332700 & 4.385824 \\
\hline $\mathrm{H}$ & 0.809290 & 4.080573 & 4.5 \\
\hline $\mathrm{H}$ & 4.44 & 0.81 & 3.897322 \\
\hline $\mathrm{H}$ & 2.176366 & -0.037674 & 8419 \\
\hline $\mathrm{H}$ & -6.034615 & 10.150883 & -3.116778 \\
\hline $\mathrm{H}$ & 3.726933 & -0.306399 & 5.875512 \\
\hline $\mathrm{H}$ & -1.593200 & 3.245950 & 4.787038 \\
\hline $\mathrm{H}$ & -2.370865 & -1.593839 & 4.330123 \\
\hline $\mathrm{H}$ & 0.211375 & -0.730027 & 6.773767 \\
\hline $\mathrm{H}$ & -0.9 & -1.937252 & 6.227544 \\
\hline $\mathrm{H}$ & 0.638967 & 1.286741 & 6.855241 \\
\hline $\mathrm{H}$ & 0.046870 & 2.865451 & 6.416299 \\
\hline $\mathrm{H}$ & 6.431678 & 2.746550 & 1.571218 \\
\hline $\mathrm{H}$ & 6.273578 & 2.188786 & 3.260659 \\
\hline $\mathrm{H}$ & 5.560162 & 1.230554 & 1.927534 \\
\hline $\mathrm{H}$ & -0.281912 & -5.668551 & 3.980346 \\
\hline $\mathrm{H}$ & -1.056887 & -4.765187 & 2.642988 \\
\hline $\mathrm{H}$ & -0.174893 & -6.278282 & 2.304015 \\
\hline $\mathrm{H}$ & -4.571110 & 4.388169 & 3.095234 \\
\hline $\mathrm{H}$ & -3.825250 & 4.089953 & 4.691088 \\
\hline
\end{tabular}




\begin{tabular}{|c|c|c|c|}
\hline $\mathrm{H}$ & -2.795910 & 4.445212 & 3.271507 \\
\hline $\mathrm{H}$ & 1.284389 & -0.684776 & 1.993475 \\
\hline W & -4.124646 & 2.888042 & -0.533467 \\
\hline & -4.370020 & 2.169230 & 1.078484 \\
\hline . & -5.577579 & 1.911485 & -0.208341 \\
\hline $\mathrm{H}$ & -0.418721 & -0.179593 & 2.093372 \\
\hline $\mathrm{H}$ & 0.885845 & 1.032114 & 2.266496 \\
\hline $\mathrm{H}$ & 2.559742 & -0.352910 & -0.107654 \\
\hline $\mathrm{H}$ & 2.436369 & 1.370377 & 0.440302 \\
\hline $\mathrm{H}$ & 1.970932 & 0.899435 & -1.225532 \\
\hline $\mathrm{H}$ & 0.719431 & -1.727291 & -0.134264 \\
\hline $\mathrm{H}$ & 0.143127 & -0.741734 & -1.500984 \\
\hline $\mathrm{H}$ & -0.958371 & -1.077209 & -0.144437 \\
\hline $\mathrm{H}$ & -2.016056 & 439 & 443 \\
\hline $\mathrm{H}$ & -1.793942 & 831 & 528 \\
\hline $\mathrm{H}$ & -0.202971 & 1.64 & 719 \\
\hline $\mathrm{H}$ & 0.30 & 2.4 & 0.3 \\
\hline $\mathrm{H}$ & 11.079768 & 5219 & 52186 \\
\hline $\mathrm{H}$ & -5.033733 & -5.38 & 0.102177 \\
\hline $\mathrm{H}$ & -10.3 & -10.846055 & -2 . \\
\hline $\mathrm{H}$ & -3.3 & -4.8 & 362 \\
\hline $\mathrm{H}$ & -5.197808 & -6.436702 & -4.063672 \\
\hline $\mathrm{H}$ & -10.192272 & -9.774895 & 1.426287 \\
\hline $\mathrm{H}$ & -3.4 & 3.86 & -3.9 \\
\hline $\mathrm{H}$ & -5.6 & 3886 & 2990 \\
\hline $\mathrm{H}$ & -3.4 & 13.5 & -0.610955 \\
\hline $\mathrm{H}$ & 14.290777 & -1.177667 & -3.428938 \\
\hline $\mathrm{H}$ & -2.524922 & 11.310667 & -0.9 \\
\hline $\mathrm{H}$ & 13.5 & 904 & -0.706780 \\
\hline $\mathrm{H}$ & 15.1 & -2.9 & -1.943997 \\
\hline $\mathrm{H}$ & -6.974969 & 12.426548 & -2.816369 \\
\hline $\mathrm{H}$ & 70 & -0.2 & -4.289271 \\
\hline $\mathrm{H}$ & 11.851253 & 132 & -3.675277 \\
\hline $\mathrm{H}$ & 5.076637 & 0.05 & 9552 \\
\hline $\mathrm{H}$ & 0.9 & 1.6 & -4. \\
\hline $\mathrm{H}$ & -2.73 & -0.4 & -3.726253 \\
\hline $\mathrm{H}$ & 0.946875 & -2.622532 & -4.192857 \\
\hline $\mathrm{H}$ & 2.969285 & -0.945124 & -5.133660 \\
\hline $\mathrm{H}$ & 3.00 & 0.55 & $-4.1 \xi$ \\
\hline $\mathrm{H}$ & -2.659642 & -2.849246 & -4.639998 \\
\hline $\mathrm{H}$ & -1.174671 & -3.798400 & -4.415415 \\
\hline $\mathrm{H}$ & -1.853919 & 2.458223 & -4.848116 \\
\hline $\mathrm{H}$ & -2.752012 & 1.856421 & -3.442825 \\
\hline $\mathrm{H}$ & -8.499794 & -9.263964 & -3.215285 \\
\hline $\mathrm{H}$ & 0.779667 & 3.895635 & -1.775832 \\
\hline $\mathrm{H}$ & -8.342594 & -8.192127 & 0.949317 \\
\hline $\mathrm{H}$ & 1.238332 & 5.573620 & -2.037670 \\
\hline $\mathrm{H}$ & -0.335357 & 4.816645 & 0.308342 \\
\hline $\mathrm{H}$ & 3.189426 & -3.432011 & -1.813302 \\
\hline $\mathrm{H}$ & 4.580142 & -4.058785 & -0.924072 \\
\hline
\end{tabular}




$\begin{array}{lrrr}\mathrm{H} & 2.796905 & -3.006318 & 0.369979 \\ \mathrm{H} & -2.540882 & -4.367047 & 0.687622 \\ \mathrm{H} & -2.184807 & -3.001040 & -0.367835 \\ \mathrm{H} & -4.439300 & -2.211196 & 0.161066 \\ \mathrm{~N} & 0.578462 & 0.339349 & 0.275747 \\ \mathrm{~N} & 0.473197 & 5.252746 & -0.132622 \\ \mathrm{~N} & 3.516899 & -2.409046 & -0.039049 \\ \mathrm{~N} & -3.987925 & -2.879324 & 0.787279 \\ \mathrm{O} & -2.459395 & 0.668623 & -0.334385 \\ \mathrm{O} & -4.396969 & -0.213993 & -0.904485 \\ \mathrm{O} & -1.060722 & 2.995905 & -3.015102 \\ \mathrm{O} & -2.176650 & -3.277353 & -2.668103 \\ \mathrm{O} & 3.158709 & -1.213095 & -3.086483 \\ \mathrm{O} & 3.459507 & -3.837760 & 2.449584 \\ \mathrm{O} & 2.694710 & 4.837631 & 2.795442 \\ \mathrm{O} & -4.069103 & -0.000567 & 2.913355 \\ \mathrm{O} & 4.598990 & 3.041696 & 2.351695 \\ \mathrm{O} & 1.034499 & -4.710949 & 2.673904 \\ \mathrm{O} & -3.724294 & 2.566591 & 3.269470\end{array}$

Compound 3.

Cartesian coordinates

191

$\mathrm{E}(\mathrm{RB} 3 \mathrm{LYP})=-4339.95442547$

$\begin{array}{llll}\text { C } & 4.718098 & -10.037881 & -3.507015\end{array}$

$\begin{array}{llll}\text { C } & 4.576019 & -1.994437 & 0.045576\end{array}$

$\begin{array}{llll}\text { C } & 7.229732 & 6.934272 & -0.318445\end{array}$

$\begin{array}{llll}\text { C } & 0.308932 & -1.570351 & -0.178163\end{array}$

$\begin{array}{lllll}\text { C } & 1.738427 & -1.652044 & 0.337201\end{array}$

$\begin{array}{llll}\text { C } & 7.340448 & 9.375178 & -1.657124\end{array}$

$\begin{array}{llll}\text { C } & -0.017751 & 0.905849 & -0.417392\end{array}$

$\begin{array}{llll}\text { C } & 8.220431 & 7.861660 & 0.002247\end{array}$

$\begin{array}{llll}\text { C } & 4.481042 & -10.780306 & -2.348417\end{array}$

$\begin{array}{llll}\text { C } & -10.660672 & 2.372872 & -1.568057\end{array}$

$\begin{array}{llll}\text { C } & 3.199599 & -8.936396 & -1.446885\end{array}$

$\begin{array}{llll}\text { C } & -8.652927 & 1.978871 & -2.891029\end{array}$

$\begin{array}{llll}\text { C } & -10.825972 & 2.202689 & -3.968660\end{array}$

$\begin{array}{llll}\text { C } & -1.933720 & -0.609619 & -0.317184\end{array}$

$\begin{array}{llll}\text { C } & 3.430147 & -8.176986 & -2.606843\end{array}$

$\begin{array}{llll}\text { C } & -0.602070 & -0.201671 & 1.705941\end{array}$

$\begin{array}{llll}\text { C } & 3.901219 & -0.707341 & -0.353369\end{array}$

$\begin{array}{llll}\text { C } & 8.280765 & 9.086324 & -0.666020\end{array}$

$\begin{array}{llll}\text { C } & 3.718429 & -10.224269 & -1.319303\end{array}$

$\begin{array}{llll}\text { C } & -0.233401 & -0.656427 & -4.048985\end{array}$

$\begin{array}{llll}\text { C } & -11.438172 & 2.390089 & -2.727684\end{array}$

$\begin{array}{llll}\text { C } & 6.348339 & 8.448608 & -1.975690\end{array}$

$\begin{array}{llll}\text { C } & 1.160334 & -0.659401 & -3.883559\end{array}$

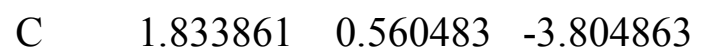




\begin{tabular}{|c|c|c|c|}
\hline $\mathrm{C}$ & 1.146185 & 1.777323 & -3.924375 \\
\hline $\mathrm{C}$ & -0.240236 & 1.759624 & -4.083713 \\
\hline $\mathrm{C}$ & -0.942473 & 0.545130 & -4.133237 \\
\hline $\mathrm{C}$ & -2.444412 & 0.534957 & -4.284261 \\
\hline $\mathrm{C}$ & 1.921983 & 3.073295 & -3.881614 \\
\hline $\mathrm{C}$ & 1.948299 & -1.947875 & -3.855073 \\
\hline $\mathrm{C}$ & -9.283810 & 2.168048 & -1.649490 \\
\hline $\mathrm{C}$ & -0.307721 & -4.938113 & -1.750517 \\
\hline $\mathrm{C}$ & 4.197082 & -8.750962 & -3.635280 \\
\hline $\mathrm{C}$ & -4.013152 & 3.092209 & -1.284480 \\
\hline $\mathrm{C}$ & 2.967125 & 3.740000 & 0.189461 \\
\hline $\mathrm{C}$ & -4.782990 & 3.137606 & 1.909561 \\
\hline $\mathrm{C}$ & -1.468632 & -5.395997 & 1.837474 \\
\hline $\mathrm{C}$ & -1.565111 & -4.968507 & 0.369722 \\
\hline $\mathrm{C}$ & -4.500021 & 2.006056 & 0.911131 \\
\hline $\mathrm{C}$ & 4.318654 & 1.373041 & 2.833208 \\
\hline $\mathrm{C}$ & 3.290282 & 2.171325 & 2.002252 \\
\hline $\mathrm{C}$ & 3.598185 & -5.787572 & -3.386003 \\
\hline $\mathrm{C}$ & 2.879998 & -6.804482 & -2.741609 \\
\hline $\mathrm{C}$ & 3.079847 & -4.499728 & -3.512533 \\
\hline $\mathrm{C}$ & 1.817190 & -4.190135 & -2.989950 \\
\hline $\mathrm{C}$ & 1.066636 & -5.190583 & -2.339273 \\
\hline $\mathrm{C}$ & 1.616492 & -6.470544 & -2.230286 \\
\hline $\mathrm{C}$ & -6.631047 & 0.875149 & -3.908411 \\
\hline $\mathrm{C}$ & -7.187219 & 1.761037 & -2.978011 \\
\hline $\mathrm{C}$ & -5.251701 & 0.683709 & -3.996058 \\
\hline $\mathrm{C}$ & -4.386192 & 1.370073 & -3.138258 \\
\hline $\mathrm{C}$ & -4.910059 & 2.273383 & -2.187468 \\
\hline $\mathrm{C}$ & -6.294234 & 2.448821 & -2.137563 \\
\hline $\mathrm{C}$ & 5.221185 & 6.222909 & -1.652857 \\
\hline $\mathrm{C}$ & 4.790275 & 6.035832 & -2.973440 \\
\hline $\mathrm{C}$ & 4.606501 & 5.443047 & -0.660618 \\
\hline $\mathrm{C}$ & 3.615617 & 4.503372 & -0.944900 \\
\hline $\mathrm{C}$ & 3.227802 & 4.316535 & -2.286612 \\
\hline $\mathrm{C}$ & 3.806210 & 5.100811 & -3.291766 \\
\hline $\mathrm{C}$ & -2.259561 & 1.150791 & 4.822438 \\
\hline $\mathrm{C}$ & -3.144688 & 1.834103 & 3.963768 \\
\hline $\mathrm{C}$ & -2.770032 & 2.972379 & 3.258182 \\
\hline $\mathrm{C}$ & -1.459669 & 3.481942 & 3.429308 \\
\hline $\mathrm{C}$ & -0.588343 & 2.817538 & 4.287176 \\
\hline $\mathrm{C}$ & -0.955895 & 1.645996 & 4.978981 \\
\hline $\mathrm{C}$ & -1.590955 & -2.293738 & 4.944038 \\
\hline $\mathrm{C}$ & -1.583670 & -3.477805 & 4.183302 \\
\hline $\mathrm{C}$ & -2.563999 & -3.773094 & 3.241054 \\
\hline $\mathrm{C}$ & -3.622523 & -2.856290 & 3.047288 \\
\hline $\mathrm{C}$ & -3.654180 & -1.697751 & 3.823605 \\
\hline $\mathrm{C}$ & -2.651551 & -1.390309 & 4.763308 \\
\hline $\mathrm{C}$ & -2.790112 & -0.085406 & 5.542996 \\
\hline $\mathrm{C}$ & 0.773512 & -1.420735 & 5.248372 \\
\hline $\mathrm{C}$ & 1.700069 & -2.281091 & 4.627355 \\
\hline
\end{tabular}




\begin{tabular}{|c|c|c|c|}
\hline $\mathrm{C}$ & 2.783350 & -1.807489 & 3.897841 \\
\hline $\mathrm{C}$ & 2.962207 & -0.408035 & 3.755562 \\
\hline $\mathrm{C}$ & 2.083847 & 0.440257 & 4.426606 \\
\hline $\mathrm{C}$ & 0.986779 & -0.037261 & 5.174266 \\
\hline $\mathrm{C}$ & 0.097278 & 0.992526 & 5.869170 \\
\hline $\mathrm{C}$ & -0.436484 & -2.063947 & 5.916868 \\
\hline $\mathrm{C}$ & -5.734921 & -2.423263 & 2.020800 \\
\hline $\mathrm{C}$ & 0.038708 & 5.295677 & 3.021509 \\
\hline $\mathrm{C}$ & 3.723093 & -3.987564 & 3.595334 \\
\hline $\mathrm{C}$ & 6.275758 & 7.211220 & -1.312941 \\
\hline $\mathrm{C}$ & -9.448737 & 2.000916 & -4.049428 \\
\hline $\mathrm{H}$ & -5.273607 & 3.982950 & 1.419750 \\
\hline $\mathrm{H}$ & -5.439305 & 2.795797 & 2.720848 \\
\hline $\mathrm{H}$ & -1.432973 & -6.485485 & 1.914518 \\
\hline $\mathrm{H}$ & -0.549633 & -4.996455 & 9252 \\
\hline $\mathrm{H}$ & -2.381427 & -5.516485 & -0.114445 \\
\hline $\mathrm{H}$ & -1.852913 & -3.897990 & 0.330849 \\
\hline $\mathrm{H}$ & -5.433122 & 1.712193 & 0.418964 \\
\hline $\mathrm{H}$ & -4.144449 & 1.124822 & 1.460240 \\
\hline $\mathrm{H}$ & 4.467161 & 1.806379 & 3.829661 \\
\hline $\mathrm{H}$ & 5.281790 & 1.378579 & 200 \\
\hline $\mathrm{H}$ & 9.053403 & 3481 & -0.4 \\
\hline $\mathrm{H}$ & 2.517547 & 1.463426 & 1.644872 \\
\hline $\mathrm{H}$ & 2.766141 & 2.898268 & 2.636922 \\
\hline $\mathrm{H}$ & -6.692097 & 3.186761 & -1.444997 \\
\hline $\mathrm{H}$ & 1.019871 & -7.23 & -1.738870 \\
\hline $\mathrm{H}$ & 4.589233 & -5.993233 & -3.779355 \\
\hline $\mathrm{H}$ & -4.162429 & 1.468669 & 3.875816 \\
\hline $\mathrm{H}$ & 0.406792 & 3.219649 & 738 \\
\hline $\mathrm{H}$ & -0.808301 & -4.216988 & 4.365540 \\
\hline $\mathrm{H}$ & -4.483778 & -1.008472 & 3.710508 \\
\hline $\mathrm{H}$ & -2.319952 & -0.180082 & 6.524082 \\
\hline $\mathrm{H}$ & 4.363140 & -8.195741 & -4.554409 \\
\hline $\mathrm{H}$ & -3.855591 & 0.074449 & 5.745313 \\
\hline $\mathrm{H}$ & 1.560284 & -3.351281 & 4.733574 \\
\hline $\mathrm{H}$ & 2.246755 & 1.511008 & 4.378853 \\
\hline $\mathrm{H}$ & -0.374223 & 0.545657 & 6.747258 \\
\hline $\mathrm{H}$ & 0.746298 & 1.785349 & 6.258959 \\
\hline $\mathrm{H}$ & -0.765537 & -1.476665 & 6.776594 \\
\hline $\mathrm{H}$ & -0.131841 & -3.035428 & 6.322735 \\
\hline $\mathrm{H}$ & -6.358095 & -2.916250 & 1.273045 \\
\hline $\mathrm{H}$ & -6.265309 & -2.401310 & 2.981023 \\
\hline $\mathrm{H}$ & -5.531119 & -1.392703 & 1.697282 \\
\hline $\mathrm{H}$ & 0.097078 & 5.560683 & 4.084642 \\
\hline $\mathrm{H}$ & 0.920608 & 4.701367 & 2.747120 \\
\hline $\mathrm{H}$ & 0.019762 & 6.205811 & 2.420045 \\
\hline $\mathrm{H}$ & 4.598878 & -4.401713 & 3.093288 \\
\hline $\mathrm{H}$ & 3.812786 & -4.144037 & 4.676825 \\
\hline $\mathrm{H}$ & 2.821906 & -4.499354 & 3.230209 \\
\hline $\mathrm{H}$ & -1.322254 & 0.584012 & 1.935206 \\
\hline
\end{tabular}




\begin{tabular}{|c|c|c|c|}
\hline $\mathrm{H}$ & 4.206479 & -2.842998 & -0.542375 \\
\hline $\mathrm{H}$ & 4.403813 & -2.199136 & 1.108141 \\
\hline $\mathrm{H}$ & 5.645667 & -1.878450 & -0.130725 \\
\hline $\mathrm{H}$ & 0.377657 & 0.081300 & 2.089837 \\
\hline $\mathrm{H}$ & -0.926882 & -1.141095 & 2.155833 \\
\hline $\mathrm{H}$ & -2.509001 & 0.319373 & -0.228450 \\
\hline $\mathrm{H}$ & -2.384720 & -1.424295 & 0.252514 \\
\hline $\mathrm{H}$ & -1.857009 & -0.877539 & -1.371121 \\
\hline $\mathrm{H}$ & -0.685215 & 1.713926 & -0.119494 \\
\hline $\mathrm{H}$ & -0.036600 & 0.793734 & -1.499464 \\
\hline $\mathrm{H}$ & 0.999966 & 1.083456 & -0.083004 \\
\hline $\mathrm{H}$ & 2.089302 & -2.651662 & 0.058553 \\
\hline $\mathrm{H}$ & 1.801899 & -1.562785 & 1.425352 \\
\hline $\mathrm{H}$ & 0.318256 & -1.611018 & -1.267744 \\
\hline $\mathrm{H}$ & -0.235918 & -2.440111 & 0.195651 \\
\hline $\mathrm{H}$ & -8.693781 & 2.128575 & -0.737475 \\
\hline $\mathrm{H}$ & 4.893782 & 5.576066 & 0.378515 \\
\hline $\mathrm{H}$ & 7.373168 & 10.328449 & -2.177920 \\
\hline $\mathrm{H}$ & 3.506445 & 4.991846 & -4.327480 \\
\hline $\mathrm{H}$ & 5.244481 & 6.611381 & -3.774682 \\
\hline $\mathrm{H}$ & 8.952845 & 7.622756 & 0.768829 \\
\hline $\mathrm{H}$ & 3.675230 & -3.747410 & -4.015462 \\
\hline $\mathrm{H}$ & 4.885476 & -11.783586 & -2.249136 \\
\hline $\mathrm{H}$ & 3.533042 & -10.791557 & -0.411172 \\
\hline $\mathrm{H}$ & -11.420127 & 2.223967 & -4.878117 \\
\hline $\mathrm{H}$ & 2.629066 & -8.505360 & -0.628563 \\
\hline $\mathrm{H}$ & -11.128234 & 2.510325 & -0.596748 \\
\hline $\mathrm{H}$ & -12.510904 & 2.549038 & -2.665022 \\
\hline $\mathrm{H}$ & 5.301499 & -10.464783 & -4.318151 \\
\hline $\mathrm{H}$ & -7.281433 & 0.309388 & -4.568950 \\
\hline $\mathrm{H}$ & -8.980147 & 1.886919 & -5.023065 \\
\hline $\mathrm{H}$ & -4.868778 & -0.010016 & -4.735151 \\
\hline $\mathrm{H}$ & -0.762881 & -1.603828 & -4.112584 \\
\hline $\mathrm{H}$ & 2.909386 & 0.575899 & -3.644108 \\
\hline $\mathrm{H}$ & -0.786881 & 2.696103 & -4.161538 \\
\hline $\mathrm{H}$ & -2.754835 & 1.031401 & -5.214560 \\
\hline $\mathrm{H}$ & -2.806106 & -0.500609 & -4.320663 \\
\hline $\mathrm{H}$ & 2.829571 & 2.972591 & -4.490303 \\
\hline $\mathrm{H}$ & 1.326979 & 3.899446 & -4.294546 \\
\hline $\mathrm{H}$ & 2.080305 & -2.338929 & -4.874304 \\
\hline $\mathrm{H}$ & 2.946940 & -1.758493 & -3.441058 \\
\hline $\mathrm{H}$ & 5.605353 & 8.696486 & -2.728910 \\
\hline $\mathrm{H}$ & -0.625856 & -3.903119 & -1.952054 \\
\hline $\mathrm{H}$ & 7.209086 & 5.973046 & 0.187923 \\
\hline $\mathrm{H}$ & -1.044666 & -5.587724 & -2.241727 \\
\hline $\mathrm{H}$ & 0.467144 & -4.819265 & 0.140598 \\
\hline $\mathrm{H}$ & -3.132427 & 3.421231 & -1.844760 \\
\hline $\mathrm{H}$ & -4.563890 & 3.998893 & -0.986917 \\
\hline $\mathrm{H}$ & -2.809839 & 2.948779 & 0.341925 \\
\hline $\mathrm{H}$ & 2.546944 & 4.461765 & 0.905439 \\
\hline
\end{tabular}




$\begin{array}{lrrr}\mathrm{H} & 2.118695 & 3.155420 & -0.198282 \\ \mathrm{H} & 4.353355 & 2.253538 & 0.272397 \\ \mathrm{~N} & -0.536223 & -0.366043 & 0.210349 \\ \mathrm{~N} & -0.320047 & -5.274156 & -0.319920 \\ \mathrm{~N} & -3.499058 & 2.346323 & -0.110422 \\ \mathrm{~N} & 3.931611 & 2.914123 & 0.926930 \\ \mathrm{O} & 2.528077 & -0.639091 & -0.286999 \\ \mathrm{O} & 4.479295 & 0.275328 & -0.751682 \\ \mathrm{O} & 1.261640 & -2.925955 & -3.068143 \\ \mathrm{O} & 2.275780 & 3.348836 & -2.526794 \\ \mathrm{O} & -3.018157 & 1.225697 & -3.167393 \\ \mathrm{O} & -3.571839 & 3.699652 & 2.421218 \\ \mathrm{O} & -2.618231 & -4.963263 & 2.562703 \\ \mathrm{O} & 3.999740 & -0.021303 & 2.961657 \\ \mathrm{O} & -4.545213 & -3.194468 & 2.103982 \\ \mathrm{O} & -1.169548 & 4.609606 & 2.725317 \\ \mathrm{O} & 3.709346 & -2.601698 & 3.276042\end{array}$

\section{$\underline{\text { References }}$}

1 Hibbert D. B.; Thordarson D. B. Chem. Commun. 2016, 52, 12792-12805. 